1 Running Title: rgsCaM granule formation and autophagy

2

3 "Corresponding author: Daniel M. Roberts, BCMB Dept., The University of Tennessee,

4 Knoxville, TN, USA Phone: 1-865-974-4070, Fax: 1-865-974-6306, e-mail: drobert2@utk.edu 


\title{
Regulator of Gene Silencing-Calmodulin associates with mRNA granules and the autophagy protein ATG8
}

\author{
W. Craig Conner, Ansul Lokdarshi, and Daniel M. Roberts
}

Department of Biochemistry \& Cellular and Molecular Biology, The University of Tennessee, Knoxville, TN 37996 USA

Summary Statement: A calcium sensor involved in the regulation of gene silencing by autophagy associates with hypoxia induced RNA granules and may serve as a cargo receptor through binding autophagy related protein 8.

Author Contributions W.C.C. designed and executed the majority of the experiments, and cowrote the manuscript with D.M.R. A.L. participated in early experimental planning and assisted with some of the preparation of materials. D.M.R., supervised and conceived the project, was involved in the design of experiments, analysis and interpretation of the data, and co-wrote the manuscript with W.C.C.

Funding information This work was supported by National Science Foundation Grant MCB1121465.

Corresponding author email drobert2@utk.edu 


\section{Abstract}

Regulator-of-gene-silencing calmodulins (rgsCaM) represent a phylogenetic subfamily of calmodulin-like calcium sensors that are targets of viral induced suppression of posttranscriptional gene silencing by secondary siRNAs. The present work shows that a stress (hypoxia) that induces mRNP granule formation also induces the relocalization of rgsCaM to cytosolic granule-like foci that interact with the surface of stress granule and processing body

42 structures. Co-expression of rgsCaM and its binding protein Suppressor of Gene Silencing 3

43 causes re-localization and integration of rgsCaM into stress granule structures. RgsCaMs

44 contain a conserved topology that consists for four EF hand like domains (three functional and

45 one divergent) that are separated into two calcium binding lobes with an extended amino

46 terminal region. RgsCaM also contains an "ATG8 family interacting motif" (AIM) within its

47 amino-terminal domain that is characteristic of selective autophagy cargo receptors. Co-

48 localization experiments and ratiometric BiFC analyses in Nicotiana benthamiana support the

49 hypothesis that rgsCaM binds directly to ATG8e through this conserved AIM domain, and the

50 two proteins co-localize with mRNP granule markers. Previous reports show that rgsCaM

51 mediates the suppression of gene silencing, at least in part, via turnover of SGS3 via

52 autophagy. A model is proposed for rgsCaM-like proteins as potential mediators of selective

53 autophagy of RNA granules in response to biotic and abiotic stresses. 


\section{Introduction}

"Regulator of Gene Silencing Calmodulin" (RgsCaM) is a calmodulin-like EF hand

protein that was originally identified in Nicotiana tabcum as a binding target of the potyviral RNA

59 silencing (VSR) protein "helper component proteinase" (HC-Pro), (Anandalakshmi et al., 2000).

HC-Pro assists in viral evasion of the host defense response by suppressing post transcriptional gene silencing (PTGS) mediated by small interfering RNA silencing pathways of the plant host (Mallory et al., 2001). Transgenic expression of HC-Pro induces the expression of rgsCaM, and rgsCaM directly binds to HC-Pro by yeast two-hybrid analysis (Anandalakshmi et al., 2000). reverses PTGS (Anandalakshmi et al., 2000). It was proposed that rgsCaM is an endogenous suppressor of PTGS, and that viral HC-Pro may take advantage of this physiological function of rgs CaM to suppress antiviral gene silencing and promote escape from the host defense response (Anandalakshmi et al., 2000).

Since its discovery as a target for potyviral HC-Pro, additional studies show that rgsCaM-like proteins interact with multiple viral suppressor proteins from a wide array of plant viruses (Nakahara et al., 2012; Li et al., 2014; Yong Chung et al., 2014; Li et al., 2017). By using surface plasmon resonance analysis, (Nakahara et al., 2012) demonstrated the interaction of tobacco rgs CaM with the bromoviral suppressor of silencing $2 \mathrm{~b}$ protein as well as other viral suppressors. Moreover, $N$. benthamiana rgsCaM is induced by the DNA geminivirus suppressor of silencing, $\beta C 1$, and is essential for conferring the suppressor activity of this viral protein (Li et al., 2014). Moreover, rgsCaM exhibits endogenous suppressor activity on its own upon overexpression in N. benthamiana (Li et al., 2014). Similarly, the Arabidopsis rgsCaM homolog, CML39 is induced by the DNA geminivirus tomato golden mosaic virus (TGMV) suppressor of silencing AL2, and interacts directly with the AL2 protein (Yong Chung et al., 
CML39 expression exhibited reduced infectivity (Yong Chung et al., 2014). Collectively, these studies indicate that rgsCaMs may be a common target for viral proteins in mediating PTGS suppressor activity, as well as serving as endogenous regulators of PTGS.

A growing body of evidence suggests that rgsCaM mediates its biological effects by targeting key RNA silencing proteins for degradation by autophagy (Yang et al., 2019).

Autophagy is a cytosolic process in which bulk cytosol, selected cargo, or organelles are engulfed in a double membrane and fused to the vacuole/lysosome for degradation and recycling of critical resources during cellular stress (Stolz et al., 2014; Yang and Bassham, 2015). The first connection of rgsCaM to autophagy was provided by (Nakahara et al., 2012) who showed that autophagic inhibitors and $\mathrm{RNA}_{\mathrm{i}}$ suppression of Autophagy-related gene 6 (ATG6) blocked cellular turnover of rgsCaM protein as well as HC-Pro and other viral suppressors of gene silencing.

A strong connection of rgsCaM to autophagy has been discovered by Li et al. (2017), who demonstrated that the SGS3 protein encoded by SUPPRESSOR OF GENE SILENCING 3 is a direct interaction target for rgsCaM (Li et al., 2017). SGS3 is an RNA-binding homodimeric protein that forms a stable complex with RNA-dependent RNA polymerase 6 (RDR6). SGS3 is an essential chaperone protein for RDR6-catalyzed synthesis of dsRNA templates required for secondary siRNA production in viral and transgene PTGS pathways (Mourrain et al., 2000; Fukunaga and Doudna, 2009; Li et al., 2017). SGS3 and RDR6 accumulate within cytosolic foci termed siRNA bodies that are proposed to be involved in transacting siRNA biosynthesis (Elmayan et al., 2009; Kumakura et al., 2009; Jouannet et al., 2012). Overexpression of rgsCaM lowered the levels of SGS3 protein as well as the number of siRNA bodies (Li et al., 2017). This effect of rgsCaM overexpression was suppressed by autophagy inhibitors and $R N A_{i}$ reduction of the expression of key autophagy genes (Li et al., 2017). From these observations, the suppression of PTGS activity by rgsCaM was attributed to the autophagic turnover of SGS3/RDR6, which would reduce the ability of the cell to produce dsRNA for the production of 
secondary siRNAs. In support of this hypothesis, it has been observed that rgsCaM preferentially suppresses secondary siRNA silencing pathways (e.g., sense transgene and viral) and not primary silencing pathways (e.g., inverted repeat hairpin transgenes) that operate

111 independent of an RDR pathway (Nakamura et al., 2014).

The question of endogenous physiological functions of rgsCaM proteins remains open.

113 Potential insight into a role in abiotic stress responses and mRNA ribostasis comes from the

114 observation that the rgsCaM-like protein CML38 from Arabidopsis is induced during flooding

115 and hypoxia stress where it accumulates within cytosolic ribonucleoprotein structures known as

116 stress granules (SGs) (Lokdarshi et al., 2016). SGs represent non-membranous cytosolic

117 granule assemblies consisting of arrested pre-initiation mRNA complexes that accumulate

118 during various cellular stresses (Protter and Parker, 2016; Chantarachot and Bailey-Serres,

119 2018). In the present study, the association of rgsCaM with hypoxia stress-induced granule

120 structures was analyzed. Further, evidence for direct association of rgsCaM with the

121 autophagic receptor ATG8 through a canonical AIM domain is presented. The significance of

122 these observations and a model for rgsCaM and related proteins as potential mediators of

123 granule turnover by autophagy (granulophagy) under conditions of cellular stress is presented. 


\section{Results}

127 RgsCaM localizes to hypoxia-induced cytosolic foci To investigate the subcellular localization of tobacco rgsCaM, Nicotiana benthamiana leaves were transiently transfected with an expression construct consisting of a translational fusion of rgsCaM with C-terminal yellow fluorescent protein (YFP) (Fig. 1). RgsCaM accumulates in distinct cytosolic foci as well as within the nuclear compartment (Fig. 1). In previous work, it was shown that submergence of $N$. benthamiana leaf disks in aqueous media induces a hypoxic state that leads to a timedependent formation of cytosolic SGs (Weber et al. 2008; Sorenson and Bailey-Serres, 2014;

134 Lokdarshi et al., 2016). A time series of confocal microscopy images after tissue submergence 135 (Fig. 2) shows that rgsCaM initially shows a diffuse cytosolic signal and that over time the 136 protein redistributes into cytosolic foci.

$\underline{\text { RgsCaM granules are independent structures that associate at the periphery of SGs and PBs }}$ under hypoxia Given the previous observation that the rgsCaM-like protein CML38 associates with hypoxia-induced SGs (Weber et al., 2008; Lokdarshi et al., 2016), the co-localization of

140 rgsCaM with the SG marker RBP47B was investigated. $N$. benthamiana leaves were co-

141 transfected with constructs expressing rgsCaM-YFP and RBP47B-CFP and imaging was

142 performed in submerged leaf discs (Fig. 3). RBP47B accumulates within cytosolic foci

143 consistent with the appearance of SGs as well as within the nucleus (Fig. 3A), similar to

144 previous observations (Lokdarshi et al., 2016). RgsCaM cytocolic foci are mostly distinct from

145 the SG marker RBP47B (Fig. 3A). This is in contrast to previous analyses of Arabidopsis

146 CML38 (Lokdarshi et al., 2016), but is supported by co-localization quantification (Manders et 147 al., 1992), that showed low Manders' co-efficients for RBP47B overlapping with rgsCaM $148\left(\mathrm{MCC}_{\mathrm{RBP} 47 \mathrm{~B}: \mathrm{rgsCaM}}=0.12\right)$, as well as for rgsCaM overlapping with RBP47B $\left(\mathrm{MCC}_{\mathrm{rgsCaM}: \mathrm{RBP} 47 \mathrm{~B}}=\right.$ 149 0.18).

However, a closer examination of the SG structures showed the apparent association of 151 smaller rgsCaM foci at the periphery of SGs (Fig. 3B). A line plot profile (Fig. 3C) shows that 
152 while the rgsCaM and RBP47B fluorescence intensity maxima are clearly distinct, in some 153 cases the two particles appear to associate with partially overlapping fluorescent signals. A 154 three-dimensional model was generated from the volumetric signal intensities in the Z-stack 155 using Imaris software (Fig. 3D, Fig. S1). The resulting model suggests that rather than 156 localizing within SGs, rgsCaM appears to interact with the surface of SGs. A similar analysis was performed with rgsCaM and the processing body (PB) marker 158 decapping protein 1 (DCP1) (Fig. 4). Leaf disc submergence resulted in the typical localization 159 of rgsCaM-YFP to small cytosolic foci, with additional apparent co-localization with larger DCP1160 CFP PB foci (Fig. 4). This dual localization of rgsCaM is reflected by Manders co-localization 161 analysis The $\mathrm{MCC}_{\mathrm{DCP} 1 \text { :rgsCam }}$ for $\mathrm{DCP} 1$ overlapping with rgs $\mathrm{CaM}$ is 0.96 , while the $162 \mathrm{MCC}_{\mathrm{rgsCaM}: \mathrm{DCP} 1}$ for rgsCaM overlapping with $\mathrm{DCP} 1$ is 0.08 . This observation suggests that most 163 PBs are associated with rgsCaM bodies, but the majority of rgsCaM bodies are independent 164 from DCP1-containing processing bodies. A closer examination at high resolution with deconvolution reveals substructure to the rgsCaM/DCP1 aggregates, and distinct localization of rgsCaM bodies and DCP1 stained PB (Fig. 5). Three-dimensional modeling using Imaris

167 through a representative PB show that rgsCaM bodies appear to a form a ring that surrounds 168 the PB (Fig. 5D, Fig. S2).

PBs are highly dynamic structures that interact and dock with other mRNP structures

170 (e.g., SG) within the cytosol, an event that is proposed to facilitate the transfer of mRNA and 171 other components between these two structures (Protter and Parker, 2016). The dynamic 172 nature of the association of rgsCaM bodies with PBs is apparent from the results of live-cell 173 confocal microscopy that show rgsCaM-YFP granules move to, dock, and stably forming a 174 physical association with DCP1 PBs (Fig. S3). These findings suggest that rgsCaM bodies 175 traffic to SGs and PBs and become bound at the surface, accumulating during hypoxia. 


\section{SGS3 localizes to hypoxia-induced cytosolic foci and colocalizes with rgsCaM Previous} observations show that rgsCaM associates with the protein product of Suppressor of Gene Silencing 3 (Li et al., 2017). SGS3 associates with cytosolic granule structures referred to as SGS3/RDR6 bodies (Kumakura et al., 2009), or siRNA bodies (Jouannet et al., 2012). To investigate further the localization of SGS3, and the relationship of these bodies to rgsCaM structures, SGS3 was expressed as a CFP fusion in $N$. benthamiana. While a small number of SGS3 granules were present at 10 min after slide preparation, the SGS fluorescence signal was largely diffuse (Fig. 6). Upon submergence, the SGS3 signal shows a time dependent redistribution to well-defined cytosolic foci (Fig. 6). These observations show that SGS3 granule-like structures, similar to SG and rgsCaM bodies, are induced by submergence and hypoxia.

To investigate whether rgsCaM and SGS3 co-localize within these structures, cotransfection experiments were performed (Fig. 7). SGS3 bodies are readily observed earlier in the hypoxia time course whereas rgsCaM fluorescence remains diffuse (Fig. 7, top panel). However, over time, rgsCaM redistributes to SGS3 granule structures (Fig. 7, bottom panel) with high Manders co-efficients of co-localization $\left(\mathrm{MCC}_{\mathrm{SGS} 3 \text { :rgsCaM }}=0.87\right.$, while the $\left.M_{\text {rgsCam:SGs3 }}=0.95\right)$. High resolution imaging with the Leica Lightning de-convolution system revealed the rgsCaM signal is virtually superimposable with the SGS3 signal, with a slightly higher rgsCaM intensity calculated at the periphery of the granule (Fig. 8B-C). Taken together, the data show that co-expression of rgsCaM with SGS3 results in a time-dependent colocalization of the two signals to granule-like structures.

SGS3 co-localizes with SG To investigate the potential relationship of SGS3 bodies that form during hypoxia and PB and SG, co-localization experiments were performed with DCP1 and RBP47B markers. Co-transfection of SGS3 with DCP1 results in the formation of cytosolic granule structures that are distinct (Fig. S4) and show low co-localization correlation co- 
201 efficients $\left(\mathrm{MCC}_{\mathrm{DCP} 1: \mathrm{SGS} 3}=0.11\right.$ and $\left.\mathrm{MCC}_{\mathrm{SGS3}: \mathrm{DCP} 1}=0.12\right)$. This is consistent with previous

202 reports that showed that SGS3 bodies are distinct from processing bodies (Kumakura et al.,

203 2009; Jouannet et al., 2012). In contrast, co-transfection of SGS3 with the SG marker RBP47B

204 shows strong co-localization (Fig. 9 A-C) with Manders co-localization co-efficients close to

205 complete superimposition $\left(\mathrm{MCC}_{\mathrm{RBP} 47 \mathrm{~B}: \mathrm{SGS} 3}=0.94\right.$ and $\left.\mathrm{MCC}_{\mathrm{SGS3}: \mathrm{RBP} 47 \mathrm{~B}}=0.88\right)$. This suggests

206 that SGS3 bodies and hypoxia-induced SG aggregates may be the same structures. This is

207 supported by previous observations that heat stress also induces the strong relocalization of

208 AGO7 and SGS3 to cytosolic granule structures (Jouannet et al., 2012).

A comparison of the rgsCaM co-localization analyses of SGS3 and RBP47B (Fig. 3 and

210 Fig. 8) reveals that while rgsCaM shows strong correlation with SGS3 bodies, co-localization

211 within the RBP47B SG marker is infrequent with a low level of rgsCaM granule-like structures

212 bound peripherally to SGs (Fig. 3). Based on the previous finding that SGS3 is a direct

213 interaction target for rgsCaM (Li et al., 2017), and the observation here that rgsCaM appears to

214 be recruited to SGS3 bodies over time (Fig. 7), it was hypothesized that overexpression of

215 SGS3 promotes the association of rgsCaM with SGs structures. To test this hypothesis, a triple

216 localization experiment was performed in which $N$. benthamiana cells were co-transfected with

217 expression constructs for SGS3-CFP along with rgsCaM-YFP and RBP47B-RFP. The results

218 show strong co-localization of all three fluorescent signals within RBP47B SGs (Fig. 9D,E).

219 These observations indicate that overexpression of SGS3 causes rgsCaM to localize strongly to

220 SGs.

221 RgsCaM interacts with the Autophagy Receptor protein ATG8e Previous studies have shown

222 that rgsCaM-dependent turnover of binding targets such as SGS3 (Li et al., 2017) and viral

223 suppressors of gene silencing (Nakahara et al., 2012) is mediated by autophagy. To investigate

224 whether rgsCaM co-localizes to autophagosomes, colocalization experiments were performed

225 with the autophagy-related gene product 8e (ATG8e) (Fig. 10). ATG8, similar to its mammalian 
counterpart LC3, is an ubiquitin-like protein that is conjugated to the amino groups of phosphatidyl ethanolamine during the biogenesis of the autophagosome. ATG8 also serves as an adaptor protein that recruits autophagic cargo to the phagophore during selective autophagy (Stolz et al., 2014; Kellner et al., 2017). As such, fluorescent protein fusions of ATG8e have served as an excellent marker for autophagosomes in plants (Contento et al., 2005). Coexpression of YFP-ATG8e and rgsCaM-CFP in N. benthamiana resulted in strong colocalization of the two proteins (Fig. 10A) with high calculated Manders co-efficients $\left(\mathrm{MCC}_{\mathrm{ATG} \text { e }: \mathrm{rgsCaM}}=0.83\right.$ and $\left.\mathrm{MCC}_{\text {rgsCaM:ATG8e }}=0.84\right)$.

ATG8 proteins recruit cargo destined for selective autophagy by interaction with an "ATG8 interacting motif" (AIM) consisting of a four amino acid consensus motif [aromatic FWY]Xaa-Xaa-[aliphatic LIV] on cargo receptor proteins (Birgisdottir et al., 2013; Kellner et al., 2017). Examination of the rgsCaM amino acid sequence revealed a potential AIM motif (residues 1519: FSRL) within the amino-terminal extension domain that precedes the first EF hand calcium binding site (Fig. S5). To investigate whether rgsCaM and ATG8 physically interact, ratiometric $\mathrm{BiFC}(\mathrm{rBIFC})$ was conducted with a single plasmid construct (pBiFC NC ATG8e, rgsCaM) containing ATG8e as an amino terminal translational fusion with the $\mathrm{N}$-terminal fragment of YFP and rgsCaM as a carboxyl-terminal translational fusion with the C-terminal fragment of YFP. The $\mathrm{pBiFC}$ plasmids also encode a constitutive fluorescent reporter protein RFP1 that allows identification of transfected cells, as well as BiFC quantitation and standardization by computing the ratio of the BiFC and RFP1 signals (Grefen and Blatt, 2012).

Transfection of $N$. benthamiana with pBiFC NC ATG8e, rgsCaM showed a robust BiFC signal (Fig 10 and Fig S6) that suggests that the two proteins interact in planta. As a negative control to test the specificity of this interaction, BiFC experiments were conducted in which rgsCaM was replaced with Arabidopsis calmodulin 2 (CaM2). CaM2 is a conventional calmodulin (i.e., not in the rgsCaM-like clade). When expressed in N. benthamiana, CaM2 
251

252

253

254

255

256

257

258

259

260

261

262

263

264

265

266

267

268

269

270

271

272

273

274

275

276

accumulates as a diffuse cytosolic signal that is distinct from cytosolic granules (Fig. S7).

Transfection of $N$. benthamiana with pBiFC NC ATG8e, CaM2 resulted in the near complete absence of the BiFC signal with the signal intensity less than $10 \%$ of the rgsCaM-ATG8e pair (Fig. 10).

To investigate further whether rgsCaM has the properties of an AIM cargo receptor, a series of site-directed mutant versions of ATG8e were examined. To disrupt the ability of ATG8e to interact with the AIM domain of proteins, a substitution of leucine 52 with alanine was generated. This leucine residue is conserved in LC3 and ATG8 proteins, and forms part of the W hydrophobic site that interacts with the first aromatic residue in the AIM motif (Noda et al., 2010). The L52A substitution resulted in the loss of the BiFC signal suggesting that an intact AIM interacting site in ATG8e is necessary for rgsCaM interaction (Fig. 10B). Another ATG8e mutant (G118A) mutation also resulted in the loss of the BiFC signal (Fig. 10B). This substitution at the C-terminus abrogates ATG8e lipidation, a process that is critical for localization of the protein to the growing phagophore membrane (Stolz et al., 2014; Marshall and Vierstra, 2018). Overall, the data suggest that the integrity of the AIM interaction site and ATG8 lipidation are essential for rgsCaM interaction.

To test whether the putative AIM motif within the amino terminal domain of rgsCaM is necessary for its interaction with ATG8, rBiFC experiments were conducted with an rgsCaM Nterminal truncation. $\Delta \mathrm{N}$-rgsCaM was engineered to remove the $\mathrm{N}$-terminal amino acids 1-42 without disruption of the EF hands within the calcium-sensor domain (Fig. S5). $\Delta \mathrm{N}$-rgsCaM fails to produce an rBIFC signal (Fig. 10B) supporting the requirement of the $\mathrm{N}$-terminal extension of rgs CaM for interaction with ATG8. To narrow down the region necessary for interaction, two site-directed mutants of rgsCaM within the putative AIM domain were tested. The F15A mutant has a substitution of a phenylalanine to alanine in the $\mathrm{W}$ region of the putative AIM domain, whereas the S16A mutant has a substitution of an alanine for a serine at a position adjacent to the aromatic residue that is conserved in all rgsCaM sequences within the Solanaceae (Fig. 
277 S5). Both substitutions result in loss of interaction with ATG8e (Fig 10B) indicating that the

278 integrity of the putative AIM domain is necessary for ATG8 interaction. Analysis of the

279 subcellular localization of the two AIM domain mutants (Fig. S8) show that they are identical to

280 wild type rgsCaM in their ability to localize to hypoxia-induced granules. This suggests that

281 disruption of the N-terminal AIM domain selectively affects association with ATG8 but not

282 assembly into hypoxia-induced bodies.

283 The data suggests that the amino-terminus of rgsCaM contains a bona fide AIM domain

284 that is characteristic of selective autophagy cargo receptors, and which is necessary for binding

285 to ATG8e. In light of previous findings that rgsCaM drives the autophagic turnover of SGS3 (Li

286 et al., 2017) and viral proteins (Nakahara et al., 2012), and the time-dependent association of

287 rgsCaM with the periphery of RNA granules (Fig. 3 and 4), rgsCaM may target mRNP particles

288 for autophagic turnover in a process similar to granulophagy (Protter and Parker, 2016). The

289 association of mRNPs and rgsCaM with autophagosomes was investigated further by triple

290 localization experiments utilizing high-resolution imaging with super-resolution (Leica Lightning)

291 deconvolution. DCP1-CFP PBs co-localize with rgsCaM-mScarlet and YFP-ATG8e in a

292 putative autophagosome (Fig. 11). Spatial resolution of the fluorescent signal shows co-

293 localization of ATG8e and rgsCaM with highest intensity at the PB periphery (Fig. 11). 
295

296

297

298

299

300

301

302

303

304

305

\section{Discussion}

$\underline{\text { RgsCaM localizes to independent hypoxia-induced cytosolic bodies that interact with SG and }}$

$\underline{P B}$ In response to cellular stresses and changing environmental conditions, eukaryotic organisms reprogram their protein translational machinery and mRNA resources. Within the cytosolic network, this occurs in part through the "RNA cycle" through which mRNA is partitioned between translating (polysomes) and non-translating (SG and PB) ribonucleoprotein particles (Protter and Parker, 2016; Chantarachot and Bailey-Serres, 2018; Ivanov et al., 2019). SGs are non-membranous cytosolic organelles that form under conditions in which mRNA translation is impaired or restricted. SGs consist of mRNA bound in an arrested pre-initiation (48S) complex comprised of small ribosomal subunits, translation initiation factors, and a myriad array of RNA binding proteins and other regulatory factors (Kedersha et al., 2013; Buchan, 2014; Protter and Parker, 2016). SGs are dynamic, and interact with and exchange mRNA and protein components with other mRNP complexes including PBs, cytosolic structures that contain machinery for mRNA degradation and posttranscriptional gene silencing (Kedersha et al., 2005; Buchan, 2014). SG are proposed to serve as "mRNA triage" centers that sort mRNA for various cellular fates based on cell needs and environmental cues (Anderson and Kedersha, 2008). In plants, submergence/hypoxia stress leads to the rapid decline in translating mRNA polysomes (Branco-Price et al., 2008), and the concomitant accumulation of cytosolic mRNP SG granules (Sorenson and Bailey-Serres, 2014). Among the proteins that accumulate within Arabidopsis SG during low oxygen stress is the rgsCaM-like protein CML38 (Lokdarshi et al., 2016). In the present work, it is shown that rgsCaM also relocalizes to cytosolic granule-like bodies in response to submergence stress. However, unlike CML38, rgsCaM bodies localize to structures that are smaller and distinct from SG and PB, but which localize to and dock to the surface of these structures. The identity of these rgsCaM bodies and their relationship to larger SG and PB remains to be determined. As noted below, co-expression of rgsCaM with the rgsCaM-binding protein SGS3 results in integration into the SG structure. The docking of 
321 mobile rgsCaM bodies with SGs and PBs may represent a precursor to this structure. During

322 the assembly of SG, smaller, more mobile sub-micrometric granules are trafficked on the

323 microtubule cytoskeleton to larger less mobile SGs for incorporation (Chernov et al., 2009;

324 Loschi et al., 2009; Gutierrez-Beltran et al., 2015; Jain et al., 2016).

325 Suppressor of Gene Silencing3 localizes to SG and induces rgsCaM association with these

326 structures The rgsCaM-interacting protein SGS3 also showed time-dependent localization to

327 cytosolic foci upon submergence. Previous work has demonstrated the localization of SGS3 to

328 similar structures designated "SGS3/RDR6 bodies"(Kumakura et al., 2009) or "siRNA bodies"

329 (Jouannet et al., 2012) that contain SGS3, RDR6, and AGO7. The potential relationship of

330 siRNA bodies to SG was noted by (Jouannet et al., 2012) who observed that heat stress

331 induced the strong accumulation of AGO7 and SGS3 with the SG protein UBP1 within cytosolic

332 granules. In the present work, it is shown that hypoxia induces the re-localization of SGS3 from

333 a diffuse cytosolic localization to cytosolic granules that are virtually superimposable with the

334 SG marker RBP47B. This suggests that SGS3 bodies and SGs may represent the same or

335 closely related particles.

336 Co-expression of rgsCaM with SGS3 also alters the localization pattern of rgsCaM.

337 Whereas rgsCaM is largely localized to distinct and independent cytosolic structures, co-

338 expression with SGS3 results in a re-localization and integration of rgsCaM within SGs. The SG

339 localization of rgsCaM occurs with different kinetics, with SGS3 granules forming before

340 recruitment of rgsCaM. Previous studies of SG show that these structures show a complex

341 architecture with "core" and "shell" components with different functions and stabilities within the

342 granule (Jain et al., 2016; Protter and Parker, 2016). Examination of the fluorescent signals

343 within SGs from the triple localization experiment (Fig. 9 D and E) show a distinct distribution of

344 the fluorescence signals of SGS3, and rgsCaM, with SGS3 appearing to be predominantly

345 associated with the core of the structure and rgsCaM showing a greater distribution to the 
periphery (presumably the "shell"). This localization and time-dependent recruitment of rgsCaM to SGS3 granules supports the proposal that SGS3 binds and assists in the recruitment of rgsCaM to SG. The signal that triggers re-localization of rgsCaM to SGS3 granules is not

349 known. However, since previous work shows that the calcium sensor domain containing EF

350 hands I and II is necessary for rgsCaM interaction with SGS3 (Li et al., 2017), a calcium signal 351 is a logical candidate.

352 RgsCaM associates with the phagophore protein ATG8 through an AIM domain What is the

353 significance of rgsCaM association with SGS3 and granule structures? Potential insight comes

354 from the work of (Li et al., 2014; Li et al., 2017) and the role of rgsCaM as a target of the

355 geminivirus suppression of host PTGS. Infection of N. benthamiana with geminivirus induces

356 the expression of rgsCaM which mediates the suppression of host RNA silencing by repressing

357 the expression of RDR6 (Li et al., 2014). Subsequent studies revealed that rgsCaM mediates

358 RDR6 turnover by binding to the SGS3 protein that forms a complex with RDR6 (Li et al., 2017).

359 RgsCaM-mediated turnover of SGS3 and RDR6 is suppressed by autophagy inhibitors, and it was proposed that rgsCaM interaction with SGS3 triggers autophagic turnover of SGS3:RDR6

361 (Li et al., 2017).

Autophagy is a mechanism by which cells degrade and recycle material from the cytosol

363 in response to developmental cues, stresses (unfolded protein response, oxidative stress, heat

364 stress, salt/osmotic stress, hypoxia), and nutrient availability (Marshall and Vierstra, 2018).

365 Autophagy involves a specialized membranous structure called a phagophore, which expands

366 and fuses around its cargo, enveloping it within a sealed double membrane autophagosome

367 vesicle. Autophagosome biogenesis is a complex process involving the coordinated interaction

368 of an array of "autophagy related proteins" (ATGs). Among these is ATG8, a ubiquitin-like

369 protein, that plays a central role in selective autophagy by recruiting specific cargo to the

370 growing phagophore (Michaeli et al., 2016; Johansen and Lamark, 2019). During 
371 autophagosome biogenesis, ATG8 is cleaved near its C-terminus by ATG4 peptidase to expose

372 a C-terminal glycine that leads to covalent conjugation ("lipidation”) to phosphatidyl

373 ethanolamine within the phagophore membrane (Stolz et al., 2014; Michaeli et al., 2016;

374 Marshall and Vierstra, 2018). ATG8 then recruits cargo to the growing phagophore through

375 "cargo adaptor proteins" that contain an ATG8-interacting motif (AIM) (Johansen and Lamark, 376 2019).

377 The present study presents evidence that rgsCaM contains an AIM domain within its 378 amino terminal domain that is conserved within rgsCaM-like proteins in the Solanaceae family

379 (Fig. S5). Ratiometric BiFC shows that ATG8e and rgsCaM physically interact, and that

380 mutations within the AIM domain abolished this interaction. Conversely, ATG8e substitutions

381 that disrupt the interaction site for AIM domain interaction or its ability to be lipidated, also

382 eliminate ATG8e:rgsCaM interaction.

383

384

385

386

387

388

389

390

391

392

393

394

$\underline{\text { RgsCaM and related proteins as targets for autophagy during viral infection and abiotic stress }}$

Taken together with previous evidence that rgsCaM mediates the autophagic turnover of SGS3 and RDR6 (Li et al., 2017), and the time dependent recruitment of rgsCaM to SGS3 granules observed here, it is proposed that rgsCaM may serve as a cargo adaptor protein that mediates the turnover over of SGS3-associated mRNP granules. This process would be similar to "granulophagy", a selective autophagy process that the leads to the degradation of SG, PB, and other RNA granules (Buchan et al., 2013; Frankel et al., 2017). Granulophagy was first discovered in yeast, but also is widely observed in metazoan cells, and defects in this process has been linked to disease states such as Amyotrophic Lateral Sclerosis (Buchan et al., 2013; Krisenko et al., 2015; Monahan et al., 2016; Chitiprolu et al., 2018). While the autophagic degradation of RNA granules in plants is less well studied, granulophagy has been proposed as part of the plant host's defense response during viral infection (Hafren et al., 2018), and in 
395

396

general has been postulated to be a component of plant RNA quality control (Yoon and Chung, 2019).

A potential mechanism for rgsCaM and related proteins in the regulation of autophagy and RNA in response to viral infection and abiotic stresses is shown in Fig. 12. With respect to abiotic stress, the expression of rgsCaM-like proteins are induced by a variety of environmental stresses, including osmotic and salinity stress, wounding, and flooding/hypoxia (Vanderbeld and Snedden, 2007; Xu et al., 2011; Tadamura et al., 2012; Lokdarshi et al., 2016). For example, the Arabidopsis rgsCaM-like protein CML38 is a core-hypoxia response protein that is induced over 300-fold during flooding or hypoxia stress, and which accumulates within SGs (Lokdarshi et al., 2016). SGs are induced during sustained hypoxia (Sorenson and Bailey-Serres, 2014) due to the decrease in translating polysomes (Branco-Price et al., 2008) associated with the energy crisis induced by anaerobic stress. CML38-associated granules are degraded during subsequent return to normoxic conditions (Lokdarshi et al., 2016). Similar to rgsCaM (Fig. 12), CML38 has the same domain organization with two EF hands containing calcium sensor domains with an extended amino-terminal domain that contains a potential AIM site (Fig. S5). Flooding stress induces the expression of autophagy related genes (ATGs), increases the number of autophagosomes, and increases autophagic degradation (Chen et al., 2015). ATG gene mutants show increased sensitivity to hypoxia indicating that autophagy is an important component of hypoxia survival (Chen et al., 2015), and plant proteins with AIM domains are targets for selective autophagy during hypoxia [e.g., S-nitroso-glutathione reductase 1 (Zhan et al., 2018)]. While the work here shows that rgsCaM associates with hypoxia-induced granules in $N$. benthamiana, its remains unknown whether rgsCaM and related proteins are part of the hypoxia adaptive response in the Solanaceae. One observation of interest is that a number of Solanaceae rgsCaM proteins possess amino-terminal cysteine residues (Fig. S5) that are characteristic of proteins regulated by hypoxia through the $\mathrm{N}$-degron pathway (Masson et al., 2019). 
With respect to viral infection, viral suppressors of silencing (e.g., potyviral HCPro and

422 geminiviral $\beta C 1$ ), induce the expression of rgsCaM or rgsCaM-like proteins (Anandalakshmi et

423 al., 2000; Endres et al., 2010; Li et al., 2014; Yong Chung et al., 2014). SGS3 binds to the

424 calcium sensor domain of rgsCaM that contains EF hands I and II [Fig. 12, (Li et al., 2017)]. It is

425 proposed here that the AIM domain in the $\mathrm{N}$-terminal extension of rgs CaM mediates the

426 recruitment of SGS3 "marked" siRNA bodies or SGs to ATG8 within the growing phagophore

427 (Fig. 12). The subsequent degradation of the SGS3/RDR6 complex/granules via autophagy

428 limits the production of secondary viral siRNAs. Notably, SGS3 degradation via autophagy is

429 also induced by other viral protein components (e.g., the viral genome-linked protein VPg from

430 potyviruses) (Cheng and Wang, 2017), indicating multiple pathways through which viruses

431 subvert the secondary siRNA defense response of the host.

In contrast to the proposal that rgsCaM serves as a viral target for the suppression of anti-viral siRNA silencing of the plant host, an opposing view for rgsCaM as an anti-viral defense protein has also been proposed (Nakahara et al., 2012). Nakahara et al. (2012) reported that rgsCaM directly binds to viral suppressor proteins (potyviral HCPro and the cucumber mosaic viral $2 \mathrm{~b}$ protein), and that it mediates the autophagic degradation of these

437 proteins resulting in the loss of viral suppression of the host silencing defense response. These 438 apparent contradictory activities of rgsCaM could possibly be explained by its proposed role as 439 an autophagy cargo receptor, and the fact that rgsCaM is able to interact with multiple protein 440 cargos (e.g., SGS3 or viral proteins, Fig. 12A) with subsequent autophagy leading to opposing 441 effects. During potyvirus infection, HC-Pro induces and becomes localized to specialized RNA 442 granule structures (potyvirus-induced RNA granules, or PGs) that contain components of both 443 SGs and PBs, as well as viral RNA (Hafren et al., 2015; Hafren et al., 2018). Selective 444 autophagic degradation of HC-Pro, and presumably PGs, through the cargo receptor NBR1 is 445 proposed to be part of the anti-viral defense response (Hafren et al., 2018). Whether rgsCaM 446 represents a separate autophagic pathway or participates in some manner within the NBR1 
447

448

449

450

451

452

453

454

455

456

457

458

459

460

461

462

463

464

465

466

467

468

469

470

471

472

pathway is unknown. As pointed out by Hafren et al. (2018), viral modulation of the autophagy pathway is complex, with both pro-viral as well as anti-viral component. How rgsCaM distinguishes viral (e.g., HC-Pro and other viral suppressors) and host (e.g., SGS3) targets during viral infection and plant defense merits further investigation.

\section{Materials and Methods}

Molecular cloning techniques and procedures Expression constructs with translational

fluorescent protein fusions driven by the Cauliflower Mosaic Virus (CaMV) 35S promoter were generated in pEarleyGate vectors: pEG101 (C-terminal YFP), pEG102 (C-terminal CFP), pEG104 (N-terminal YFP), and pK7RWG2 (C-terminal RFP) (Karimi et al., 2005). All are Gateway-compatible destination vectors with attP1 and attP2 recombination sites. Donor clones were generated in pDONR207 (Invitrogen).

Nicotiana tabacum rgsCaM cDNA (Anandalakshmi et al., 2000) and Arabidopsis ATG8e cDNA (Contento et al., 2005) were amplified using gene specific primers that add the attB1 recombination site to the 5' end and the attB2 site to the 3' end (Supplemental Table S1). For SGS3 and DCP1 cloning, cDNA was generated with the SuperScript III first strand cDNA synthesis system (ThermoFisher) using total RNA from 1-2 week old Arabidopsis thaliana Col-0 seedlings as a template followed by amplification with gene specific primers with the attB1 recombination and the attB2 recombination sites (Supplemental Table S1). Amplified PCR products were isolated and purified by gel electrophoresis, and were recombined with the Gateway-compatible donor vector pDONR207 in a BP clonase II (Invitrogen) reaction as previously described (Lokdarshi et al., 2016). The desired cloned products were confirmed by DNA sequencing, and donor clones were recombined with one of the localization vectors above in an LR clonase II reaction per manufacturer's protocol (Invitrogen). The following Gatewaycompatible clones were generated and used in this study: pEG 101 rgsCaM-YFP, pEG 102 rgsCaM-CFP, pEG 102 DCP1-CFP, pEG 101 SGS3-YFP, pEG 102 SGS3-CFP, pEG 104 YFP- 
473

474

475

476

477

478

479

480

481

482

483

484

485

486

487

488

489

490

491

492

493

494

495

496

497

ATG8e, and pK7RWG2 RBP47B-RFP. pEG 102 RBP47B-CFP was generated as previously described in (Lokdarshi et al., 2016).

A translational fusion construct of $r g s C a M$ with the $m S c a r l e t$ fluorescent reporter protein (Bindels et al., 2017) was prepared in pBin19 (Frisch et al., 1995). Xbal and Xmal sites were introduced into the Nt-rgsCaM open reading frame at the 5' and 3' ends respectively by PCR using gene specific primers with these sites (Table S1). Nt-rgsCaM was then subcloned into these sites within a shuttle plasmid (a gift from Andreas Nebenführ, University of Tennessee Knoxville) to generate the $m$ Scarlet translational fusion under the control of the 35SCaMV promoter. A fragment containing 35S:rgsCaM-mScarlet was excised by digestion with Sacl and HindlII, and subcloned into pBin19.

pBiFCt-2in1 plasmids (Grefen and Blatt, 2012) were used for ratiometric BiFC. Donor clones were generated in pDONR 221 plasmids containing either attP1 and attP4 sites, or attP3 and attP2 sites. BiFC pairs were generated by using Multisite LR clonase II plus (Invitrogen) with each reaction containing the desired pDONR221 donor construct pairs and a pBiFC 2-in1 plasmid (3:3:1 molar ratio) (Grefen and Blatt, 2012). Site-directed mutagenesis was conducted on pDONR 221 plasmids containing Arabidopsis ATG8e and on pDONR 221 plasmids containing Nt-rgsCaM. Mutagenesis was performed according to the "round the horn" protocol (OpenWetWare). All mutagenesis primers and corresponding mutations are described in Table S1. An N-terminal truncation of rgsCaM that removes amino acids 1-42 was also generated. The coding region of Nt-rgsCaM corresponding to amino acids 43-190 was amplified from pDONR207 Nt-rgsCaM using an rgsCaM $\Delta \mathrm{N}$ attB1 forward primer and the rgsCaM attB4 reverse primer (Table S1) and was recombined into pDONR 221 to generate a donor plasmid containing $\Delta N-r g s C a M$.

Sanger DNA sequence analysis of all plasmid constructs was performed on a Applied Biosystems 3730 Genetic Analyzer at the University of Tennessee Genomics Core Facility. 

germinated in Lambert LM-GPS soil and were grown in an environmental growth chamber with long day conditions $\left(16 \mathrm{~h}\right.$ light at $25^{\circ} \mathrm{C} / 8 \mathrm{~h}$ dark at $23^{\circ} \mathrm{C}$ ) and $50 \%$ humidity under a light intensity of $100 \mu \mathrm{mol} \mathrm{m} \mathrm{m}^{-2} \mathrm{~s}^{-1}$. Plants that were between four to five weeks old (prior to flowering) were used for transfections. Binary vectors were introduced into Agrobacterium tumefaciens strain

503 GV3101 (Koncz and Schell, 1986) by electroporation (Jones, 1995) or heat shock (Hofgen and

504 Willmitzer, 1988), and selected colonies were grown at $28^{\circ} \mathrm{C}$ for $24-48 \mathrm{hr}$ in LB broth with 50

$505 \mu \mathrm{g} / \mathrm{mL}$ rifampicin, $50 \mu \mathrm{g} / \mathrm{mL}$ gentamycin and either $50 \mu \mathrm{g} / \mathrm{mL}$ kanamycin (for pEarleyGate plasmids) or $100 \mu \mathrm{g} / \mathrm{mL}$ spectinomycin (for pBiFCt-2in1 plasmids). Cells were collected by centrifugation at 3,000 $\times \mathrm{g}$ for $10 \mathrm{~min}$ and pellets were resuspended in $10 \mathrm{mM} \mathrm{MgCl}_{2}, 10 \mathrm{mM}$ MES-NaOH, pH 5.7, $200 \mu \mathrm{M}$ acetosyringone, and infiltration of $N$. benthamiana was performed as in (Brunkard et al., 2015). The inoculum was incubated with slow rotation at $20^{\circ} \mathrm{C}$ for $2-4 \mathrm{hr}$ to allow acetosyringone-induction of virulence gene expression. For co-infiltrations, cultures

511 containing each plasmid were combined prior to infiltration so that each was equivalent to $O D_{600}$

512 of 0.4 . Combined $A$. tumefaciens cultures were infiltrated into the abaxial side of leaves using a 513 needleless syringe and the infiltrated region was marked. Plants were kept at $20^{\circ} \mathrm{C}$ overnight on 514 a lab bench, and then transferred to growth chambers under standard long day conditions (16 $\mathrm{h}$ 515 light, $22-25^{\circ} \mathrm{C}, 8 \mathrm{~h}$ dark, $20-22^{\circ} \mathrm{C}$ ). Imaging experiments were performed two days after 516 infiltration. Hypoxia stress was administered by submergence of transfected $N$. benthamiana leaf 518 sections as previously described (Lokdarshi et al., 2016). One $\mathrm{cm}^{3}$ leaf discs were cut from 519 infiltrated regions adjacent to the site of injection. Tissues were placed in DI water and a brief 520 manual vacuum was administered with a syringe to remove air pockets as described by 521 (Brunkard et al., 2015). Hypoxia was administered by submerging leaf samples in DI water on a 522 slide under a \#1.5 coverslip, adaxial face down (Lokdarshi et al., 2016). Imaging was performed 
523 on adaxial epidermal cells. Except where indicated in specific figures, submergence stress was

524 administered between 1.5 to $4 \mathrm{hr}$ before imaging.

525 Microscopy and imaging techniques An Leica SP8 laser scanning confocal microscope

526 (Wetzlar, Germany) was used for all confocal microscopy performed in this study. For co-

527 localization experiments, each fluorescence channel was captured sequentially to prevent signal

528 cross-talk. Sequential imaging was performed between scan lines to limit the impact of granule

529 movement. Excitation/emission bandwidth settings for imaging were: $405 \mathrm{~nm} / 425-500 \mathrm{~nm}$

530 (DAPI), $470 \mathrm{~nm} / 480-530 \mathrm{~nm}$ (CFP), $514 \mathrm{~nm} / 540-575 \mathrm{~nm}$ (YFP), $575 \mathrm{~nm} / 600-660 \mathrm{~nm}$ (RFP) and

$531590 \mathrm{~nm} / 600-660$ (mScarlet). Chloroplast autofluorescence was captured over 680-740 nm. To

532 reduce chloroplast autofluorescence when imaging CFP, YFP or RFP, time gating was used on

533 the HyD hybrid detectors to exclude emission from the first 0.3 ns following pulsed excitation

534 from the white light laser as described by (Kodama, 2016). Laser strength and gain were

535 adjusted for each image to produce signal just below saturation.

536 For DAPI staining (4',6-diamidino-2-phenylindole; Sigma, St. Louis, MO), transfected leaf

537 discs were cut and placed in DI water with $5 \mu \mathrm{g} / \mathrm{ml}$ DAPI for 30 min prior to mounting in DI water

538 for imaging. Co-localization imaging was performed over a Z-series to include the top surface of

539 the cell. Except where indicated in specific figures, the step-size between optical sections was

540 between 1-2 $\mu \mathrm{m}$. The pinhole size was 0.8-1 AU.

541

Confocal micrographs were captured in Leica LASX software and uncompressed images

542 were exported and analyzed in ImageJ (Schneider et al., 2012). Brightness was increased to

543 just below saturation, and the built-in smoothing filter in ImageJ was applied to reduce noise.

544 ImageJ was used to add scale bars, false colorations and overlays, and to perform maximum

545 intensity 2D projections. Except where indicated in specific figures, 2D projections shown

546 represent between 20 to 27 optical sections.

547 Colocalization analysis was performed in ImageJ using the Just Another Colocalization

548 Plugin (JACoP) (Bolte and Cordelieres, 2006). Images corresponding to the two channels were 
549 imported and a region of interest was selected that corresponds to the cytosol of an individual

550 cell. JACoP was used to compute Mander's coefficients (Manders et al., 1992) to obtain

551 quantitative analyses and spatial descriptions of overlapping signals from the two fluorophores.

552 Manders' coefficients (MCC) are more informative that other co-localization techniques (e.g.,

553 Pearson correlation co-efficients) when there are unequal co-localizations, such as one protein

554 strongly co-localizing with the second, while the second also showing independent localization

555 (Bolte and Cordelieres, 2006; Dunn et al., 2011). Two Mander's coefficients were computed: the

556 first co-efficient quantifies the fraction of the signal from channel $A$ that overlaps with channel B,

557 and the second quantifies the fraction of the channel B signal that overlaps with channel A

558 (Manders et al., 1992).

559 Data for line plots were obtained by drawing a line through the indicated region in

560 ImageJ and using the built-in plot profile function. The output of brightness values by distance

$561(\mu \mathrm{m})$ for each pixel was imported into Excel, converted into percentages based on the highest

562 obtained value by channel, and then imported into Prism 8.1.1 (GraphPad) for figure generation.

563 Standard deconvolution of images was done by using the Huygens Essentials software

564 (Scientific Volume Imaging, Hilversum, The Netherlands) using the standard profile for

565 automatic deconvolution. The Leica Lightning deconvolution system was used in certain

566 experiments (triple localization Fig. 9 and 11, and rgsCaM and SGS3, Fig. 8) to optimize image

567 capture settings for Z-axis step size, resolution and scan speed, and to maximize resolution.

568 The adaptive deconvolution profile setting in the Leica Lightning Deconvolution package was

569 used.

Imaris (Oxford Instruments, Zurich, Switzerland) was used to generate 3D models from

571 deconvolved Z-series signal volumes in selected co-localization experiments. Thresholds were

572 defined for CFP and YFP signals to produce models corresponding to their respective signal

573 volume data. To correct for signal bleed in the Z-axis, the setting for the Z-axis step size in 
574 Imaris was adjusted to reflect the spherical shape of the imaged granules (rgsCaM granules

575 were the reference for Fig 3, and the DCP1 granules were the reference in Fig 4).

576 Ratiometric bimolecular fluorescence complementation For BIFC analysis, pBiFC 2-in-1

577 plasmids (Grefen and Blatt, 2012) expressing the two proteins of interest as nYFP or cYFP

578 fusions were transfected into N. benthamiana as described above. The BiFC plasmid also

579 expresses a constitutive mRFP1 that serves as a reference for ratiometric comparison to the

580 YFP BiFC signal. This ratiometric approach allows for quantification of BiFC signals and

581 controls for differences in plasmid copy number among transfected cells. mRFP1 was imaged

582 with excitation/emission bandwidth of $543 \mathrm{~nm} / 560-615 \mathrm{~nm}$, while the eYFP BiFC signal used

$583514 \mathrm{~nm} / 520-550 \mathrm{~nm}$. Both channels used time gating to exclude the first $0.3 \mathrm{~ns}$ to reduce

584 chloroplast autofluorescence (Kodama, 2016). Sequential imaging was performed between

585 scan lines, with a line averaging of four used for each image of a single optical section. Four

586 biological replicates were used, with at least five cells analyzed from each. BiFC clones

587 generated and used in this study include: pBiFC NN ATG8e, rgsCaM; pBiFC NC ATG8e, 588 rgsCaM; pBiFC NN ATG8e, CaM2; pBiFC NC ATG8e, CaM2; pBiFC NC ATG8e-L52A, rgsCaM; $589 \mathrm{pBiFC}$ NC ATG8e-G118A, rgsCaM and pBiFC NC ATG8e, $\Delta$ N-rgsCaM; pBiFC NC ATG8e, 590 rgsCaM-F15A; pBiFC NC ATG8e, rgsCaM-S16A, where the two letter code indicates whether 591 the split-YFP is positioned at the $\mathrm{N}$ - or $\mathrm{C}$-terminus of each coding sequence. Identical BiFC 592 results were obtained for both $\mathrm{N}$ and $\mathrm{C}$ terminal fusions of rgs $\mathrm{CaM}$, and only the results with $\mathrm{C}-$ 593 terminal fusions of rgs $\mathrm{CaM}$ and rgsCaM mutants are shown. A negative control construct 594 (pBiFC NC ATG8e, CaM2) was generated in which Arabidopsis calmodulin 2 (CaM2) was used 595 in place of $\mathrm{rgsCaM}$.

Ratiometric BiFC analysis was similar to (Grefen and Blatt, 2012). In ImageJ, a region of 597 interest was traced to select individual cells. Nuclei were not visible in all images and they were 598 excluded from the ROI when present in case the BiFC complex had different nuclear preference 599 than the mRFP1. Image J was used to compute the average brightness of the ROI for YFP and 
600

601

602

603 (Graph Pad).

604

605

606

607

608

609

610

611

612

613

614

615

616

617

618

619

620

621

622

623 Knoxville.

RFP channels, this was then exported to Excel where the eYFP:mRFP1 ratio was calculated for each cell and the values were normalized to the highest signal, which in all experiments was for ATG8e and rgsCaM. Statistical analysis was performed in GraphPad Prism version 8.1.1

Gene ID and accession numbers The GenBank accession numbers for coding sequences used in this study are: NM_100710.4 (DCP1), NM_112800.4 (RBP47B), NM_001343817 (SGS3), NM_180100.7 (ATG8e), NM_180013.3 (CaM2), AF329729.1 (rgsCaM).

\section{Acknowledgements}

We thank Dr. Andreas Nebenführ and Anna Vick (University of Tennessee, Knoxville) for the kind gift of the pAN990 mScarlet plasmid, and Dr. Tessa Burch-Smith (University of Tennessee, Knoxville) for guidance on transfecting and imaging $N$. benthamiana leaves and for critical comments during the preparation of the manuscript. Microscopy was conducted at the Advanced Microscopy and Imaging Center, a core facility at the University of TennesseeKnoxville, with support from Dr. John Dunlap and Dr. Andreas Nebenführ. Automated Sanger sequencing was conducted at the Genomics Core facility at the University of Tennessee-

\section{Figure Legends}

Fig 1. Localization of rgsCaM-YFP in cytosolic granule-like foci in $N$ benthamiana leaf cells. $N$ benthamiana leaves were transfected with a rgsCaM-YFP expression construct by Agrobacterium infiltration, and were subjected to submergence-induced hypoxia as described in 
624 the Materials and Methods. RgsCaM-YFP was visualized by confocal microscopy, and nuclei

625 were stained with DAPI. Scale bars are $50 \mu \mathrm{m}$.

626 Fig 2. Timecourse of. RgsCaM-YFP cytosolic foci formation in response to submergence-

627 induced hypoxia. A representative cell from a transfected N. benthamiana leaf expressing

628 rgsCaM-YFP was imaged over the indicated times after submergence. Scale bar is $50 \mu \mathrm{m}$.

Fig 3. Co-localization of rgsCaM-YFP and stress granule markers in hypoxic $N$.

630

benthamiana leaves. Co-localization experiments with $N$. benthamiana leaves co-transfected

631 with constructs that express rgsCaM-YFP and the stress granule marker RBP47B-CFP. A) 2D

632 projections from a representative cell, consisting of 99 optical sections with a step size of 0.3 $\mu \mathrm{m}$, and subjected to standard deconvolution as described in the Materials and Methods. Scale bars are $10 \mu \mathrm{m}$.

B) Magnification of the boxed region shown in panel A. Scale bar is $1 \mu \mathrm{m}$. A smoothing filter was applied in Leica LasX software.

C) A line plot profile of fluorescence intensities calculated along the axis shown by the dashed line in panel B. D) 3D model reconstruction from the dataset shown in B using the Imaris Software (see also Fig. S1). Scale bar is $1 \mu \mathrm{m}$.

Fig 4. Co-localization of rgsCaM-YFP and processing body markers in hypoxic $N$. benthamiana leaves. Leaf sections of $N$. benthamiana leaves co-transfected with constructs that express rgsCaM-YFP and the processing body marker DCP1-CFP. Scale bar is $50 \mu \mathrm{m}$.

643 two-dimensional projection from 27 optical sections (0.3 $\mu \mathrm{m}$ step size) of a representative DCP1

644 processing body structure from a $N$. benthamiana cell co-transfected with DCP1-CFP and

645 rgsCaM-YFP as described in Fig. 4. Leaf sections were subjected to submergence-induced

646 hypoxia before imaging. The image was subjected to standard deconvolution and a smoothing

647 filter was applied in Leica LasX software. Scale bars: top panel is $5 \mu \mathrm{m}$, bottom panel is $1 \mu \mathrm{m}$. 
648

649

650

651

652

653

654

655

656

657

658

659

660

661

662

663

664

665

666

667

668

669

670

671

B) A line profile for the fluorescence intensities through the dashed line shown in panel A. C) 3D model constructed from the dataset shown in A using the Imaris Software (see also Fig. S2). Scale bar is $0.5 \mu \mathrm{m}$.

Fig 6. Time course of formation of SGS3-CFP cytosolic foci in response to submergenceinduced hypoxia. $N$. benthamiana leaves were transfected with a SGS3-CFP construct by Agrobacterium infiltration and was imaged over the indicated times after submergence. Scale bar is $50 \mu \mathrm{m}$.

Fig 7. SGS3 and rgsCaM co-localize into cytosolic granule-like structures during hypoxia with different kinetics. $N$. benthamiana leaves were co-transfected with constructs that express rgsCaM-YFP and the stress granule marker SGS3-CFP and leaf sections were subjected to hypoxia as in Fig. 6. Images are 2D projections of 6 optical sections with a step size of $2 \mu \mathrm{m}$ collected at $30 \mathrm{~min}$ and $90 \mathrm{~min}$ after submergence. Scale bars are $50 \mu \mathrm{m}$.

Fig 8. Co-localization of rgsCaM-CFP and SGS3-YFP. A) Leaves of $N$. benthamiana were co-transfected with constructs that express rgsCaM-YFP and SGS3-CFP, and leaf sections were subjected to submergence-induced hypoxia. Scale bar is $30 \mu \mathrm{m}$. B) Enlargement of the structure in A marked with the arrowhead. Images were subjected to a smoothing filter in LAS X and deconvolution was performed with the Leica Lightning system. Scale bar is $1 \mu \mathrm{m}$. C) Line plot profile of fluorescence intensities through the dashed line in B.

Fig 9. SGS3 and Stress Granule co-localization in N. benthamiana cells. A) Leaves of $N$. benthamiana were co-transfected with constructs that express SGS3-YFP and the stress granule marker RBP47B-CFP and leaf sections were subjected to hypoxia. Scale bar is $10 \mu \mathrm{m}$.
B) Magnification of the granule marked by the arrowhead in A, with a smoothing filter applied in

$$
\text { Leica LAS X. Scale bar is } 0.5 \mu \mathrm{m} \text {. C) Line plot profile though the dashed line in B. D) Triple }
$$
localization images from $N$. benthamiana leaf epidermal cells co-transfected with SGS3-CFP, 
672 RBP47B-RFP and rgsCaM-YFP. Images were taken $5 \mathrm{hr}$ after slide preparation and are 2D

673 projection of 4 optical sections with a Z-step size of $0.13 \mu \mathrm{m}$. Scale bar is $1 \mu \mathrm{m}$. The Lightning

674 deconvolution system was used and an additional smoothing filter was applied in LAS X. E) Line

675 plot through the dashed line in D.

676 Fig 10. Co-localization and bimolecular fluorescence complementation analysis rgs CaM

677 and ATG8e. A) Coexpression of rgsCaM-CFP and YFP-ATG8e in N. benthamiana leaves.

678 Images were taken from leaf sections after submergence-induced hypoxia. Scale bars is 20

$679 \mu \mathrm{m}$. B) Ratiometric BiFC quantification from $N$. benthamiana cells transfected with pBiFC

680 constructs expressing the following protein pairs (see Materials and Methods for details):

681 ATG8e, rgsCaM; ATG8e, AtCaM2; ATG8e, $\triangle \mathrm{N}$-rgsCaM; ATG8e, rgsCaM F15A; ATG8e,

682 rgsCaM S16A. C) Ratiometric BiFC quantification from $N$. benthamiana cells transfected with

683 pBiFC constructs done as in panel B, except with the following protein pairs: ATG8e, rgsCaM;

684 ATG8e, AtCaM2; ATG8e L52A, rgsCaM; ATG8e G118A, rgsCaM. In both B and C, BiFC

685 signals were standardized as a ratio to constitutive mRFP1 signals and were normalized to the

686 highest value, which was the rgsCaM, ATG8e BiFC pair. The data represent the values from

687 four biological replicates (20 to 30 cells). Error bars show standard deviation. Different letters

688 over histograms represent statistically significant differences ( $p$ values $<0.0001$ ) based on One-

689 way ANOVA analysis with multiple comparisons. 
Fig. 11 Triple co-localization analysis of rgsCaM, ATG8e, and the PB marker DCP1 in hypoxic $\boldsymbol{N}$. benthamiana leaves. A) Co-localization of DCP1-CFP, YFP-ATG8e and rgsCaMmScarlet transfected $N$. benthamiana leaves after submergence-induced hypoxia. Images are a 2D projection of 9 optical sections with a Z-step size of $0.2 \mu \mathrm{m}$. The scale bar is $1 \mu \mathrm{m}$. These images were generated using the Lightning deconvolution system. The smooth rendering filter was applied in LAS X. B) Line plot through the dashed line in panel A generated from the unfiltered data.

Fig. 12 Model for rgsCaM as a regulator of autophagic turnover of SGS3 and other cargo.

A. Top panel, domain model for rgsCaM based on homology modeling with a calciumcalmodulin template (pdb 1cll). The bilobal structure with EF hands I (white) and II (magenta) comprising the $1^{\text {st }}$ calcium binding lobe (residues 47-119) and EF hands III (yellow) and IV (green) comprising the $2^{\text {nd }}$ calcium binding lobe. The $\mathrm{N}$-terminal domain is simply shown as an extended structure with the predicted AIM domain residues shown in space filled representation. Bottom panel, diagram showing the predicted sites of binding of rgsCaM to ATG8, as well as to SGS3 and other cargo (PB and viral suppressors of gene silencing). B. Model for rgsCaM mediated turnover of SGS3, RNP granules, and other cargo. RgsCaM and rgsCaM-like proteins (e.g., CML38) are induced during viral infection and selective abiotic stresses (e.g.,

707 hypoxia). Suppressor of gene silencing 3 (SGS3) resides in siRNA bodies as well as stress 708 granule structures in complex with RNA-dependent RNA-polymerase 6 (RDR6). SGS3 recruits cytosolic rgsCaM to stress granules, and mediates the turnover (and suppression of secondary siRNA silencing) through targeting of SGS3 granules to autophagosomes (granulophagy) by

711 rgsCaM interaction with ATG8. RgsCaM also interacts with processing bodies through

712 interaction with an unknown PB protein target. In addition to degradation of the SGS3/RDR6-

713 mediated siRNA production machinery, autophagic degradation of mRNPs can recycle material

714 during biotic and abiotic stress, and may control mRNA homeostasis and expression. 


\section{Supplemental Material}

716 Fig S1. Fitting of SG-marker RBP47B and rgsCaM imaging data to 3D models.

717 Fig. S2. Fitting of PB-marker DCP1 and rgsCaM imaging data to 3D models.

718 Fig S3. RgsCaM body docking onto DCP1 processing body.

719 Fig S4. SGS3 and DCP1 colocalization in N. benthamiana leaves.

720 Fig S5. Domain structures and amino acid sequence of rgsCaM and rgsCaM-like proteins.

721 Fig S6. Bimolecular fluorescence complementation of wt and mutant forms of rgs CaM 722 and ATG8e.

723 Fig S7. Co-localization experiments with Arabidopsis CaM2 and SGS3 in N. benthamiana 724 leaves.

725 Fig S8. Localization of rgs CaM AIM mutants in N. benthamiana.

726 Table S1: PCR primers for molecular cloning, site-directed mutagenesis and sequencing 

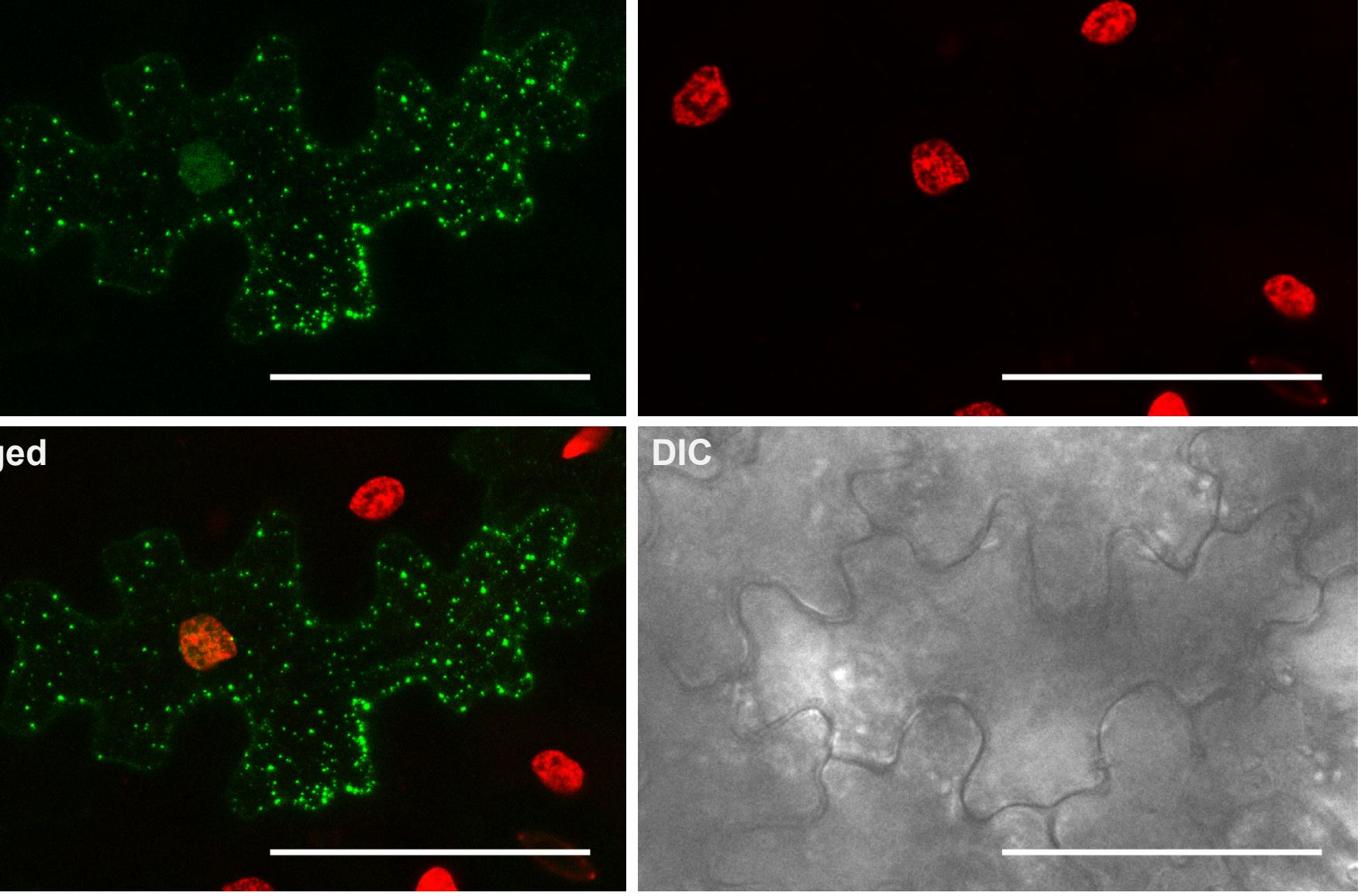

Fig 1. Localization of rgsCaM-YFP in cytosolic granule-like foci in $\mathbf{N}$ benthamiana leaf cells. $N$. benthamiana leaves were transfected with a rgsCaM-YFP expression construct by Agrobacterium infiltration, and were subjected to submergence-induced hypoxia as described in the Materials and Methods. RgsCaM-YFP was visualized by confocal microscopy, and nuclei were stained with DAPI. Scale bars are $50 \mu \mathrm{m}$. 


\section{$50 \mathrm{~min}$}

Fig 2. Timecourse of rgsCaM-YFP cytosolic foci formation in response to submergence-induced hypoxia. A representative cell from a transfected $N$. benthamiana leaf expressing rgsCaM-YFP was imaged over the indicated times after submergence. Scale bar is $50 \mu \mathrm{m}$.

\section{$70 \mathrm{~min}$}

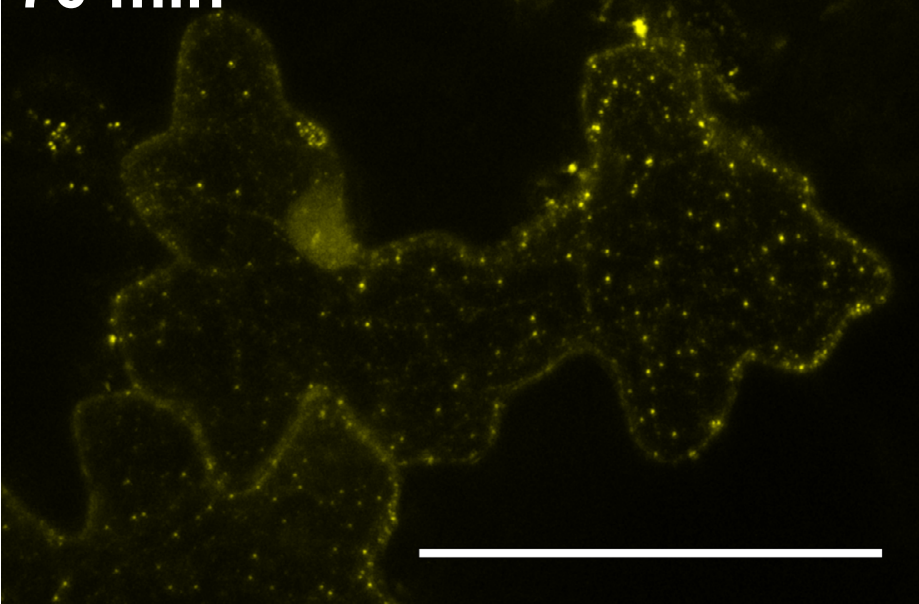



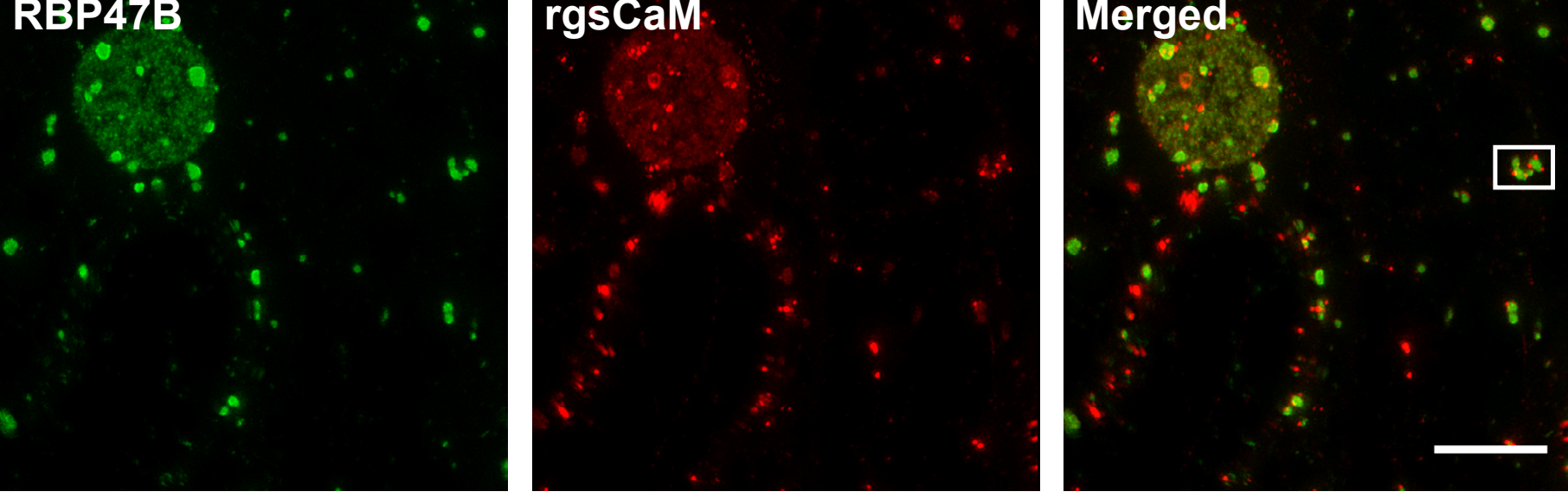

\section{B}

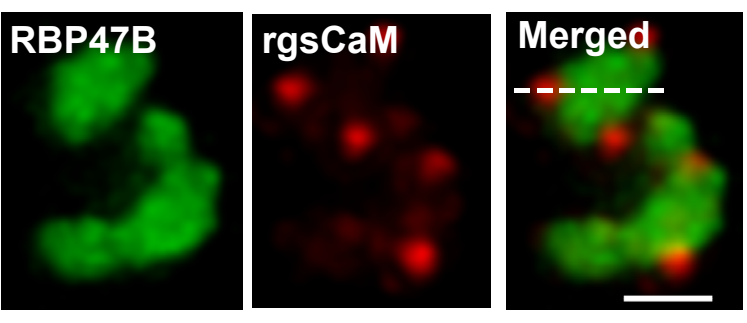

D
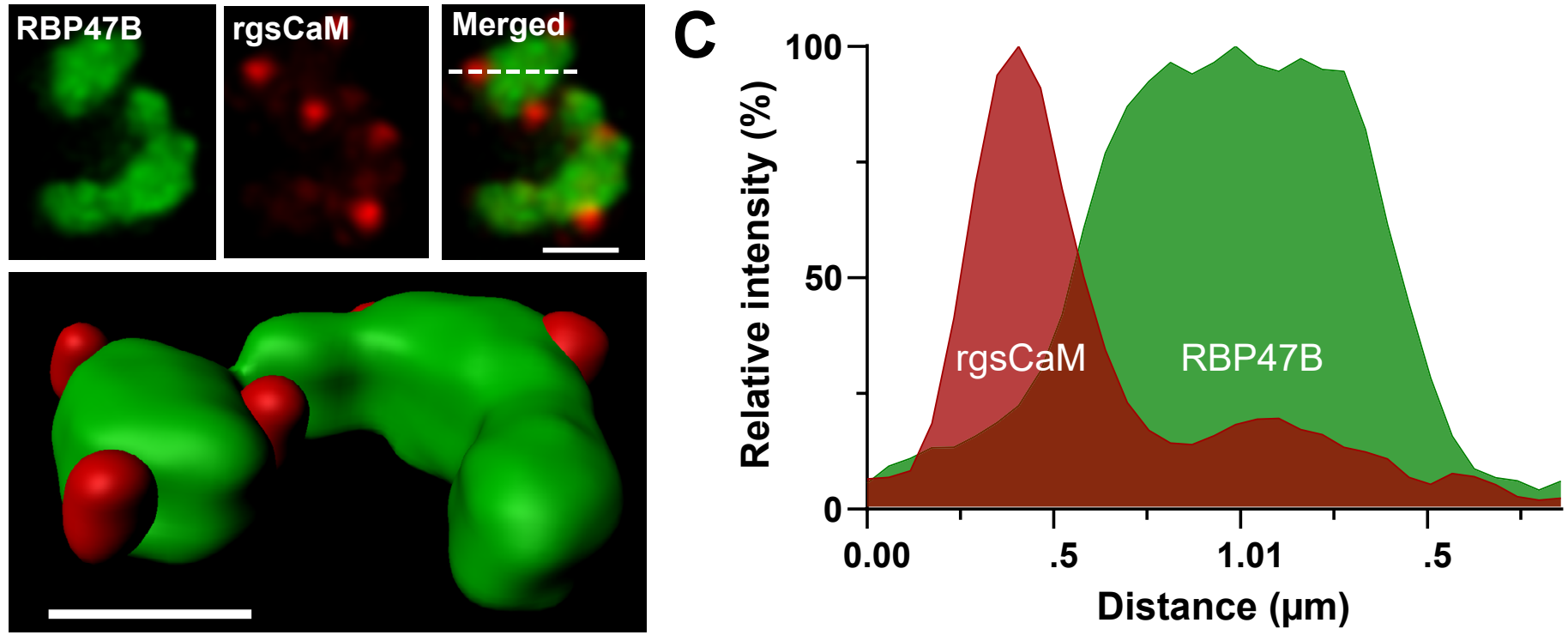

Fig 3. Co-localization of rgs CaM-YFP and stress granule markers in hypoxic $\boldsymbol{N}$. benthamiana leaves. Co-localization experiments with $N$. benthamiana leaves co-transfected with constructs that express rgsCaM-YFP and the stress granule marker RBP47B-CFP. A) 2D projections from a representative cell, consisting of 99 optical sections with a step size of $0.3 \mu \mathrm{m}$, and subjected to standard deconvolution as described in the Materials and Methods. Scale bars are $10 \mu \mathrm{m}$. B) Magnification of the boxed region shown in panel A. Scale bar is $1 \mu \mathrm{m}$. A smoothing filter was applied in Leica LasX software. C) A line plot profile of fluorescence intensities calculated along the axis shown by the dashed line in panel B. D) 3D model reconstruction from the dataset shown in B using the Imaris Software (see also Fig. S1). Scale bar is $1 \mu \mathrm{m}$. 


\section{Merged}

\section{DIC}

Fig 4. Co-localization of rgsCaM-YFP and processing body markers in hypoxic $\boldsymbol{N}$. benthamiana leaves. Leaf sections of $N$. benthamiana leaves co-transfected with constructs that express rgsCaM-YFP and the processing body marker DCP1-CFP. Scale bar is $50 \mu \mathrm{m}$. 


\section{B DCP1}

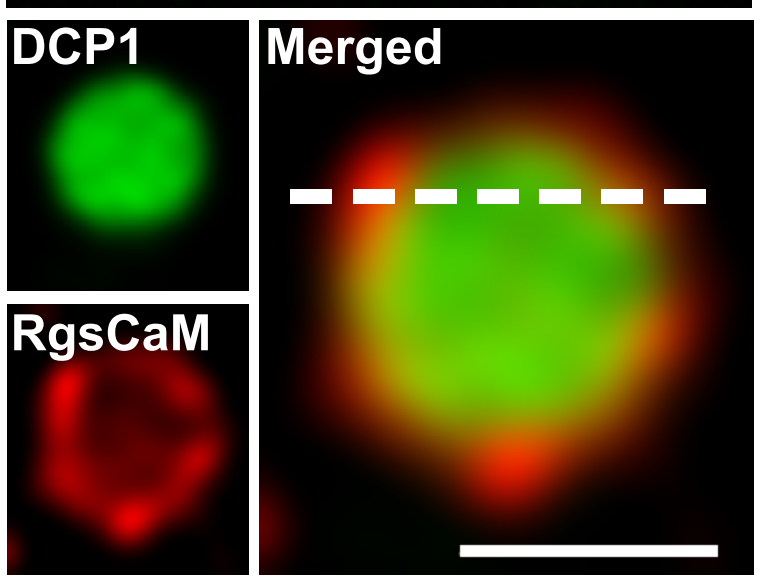

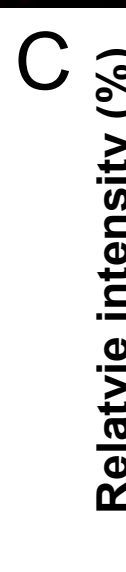
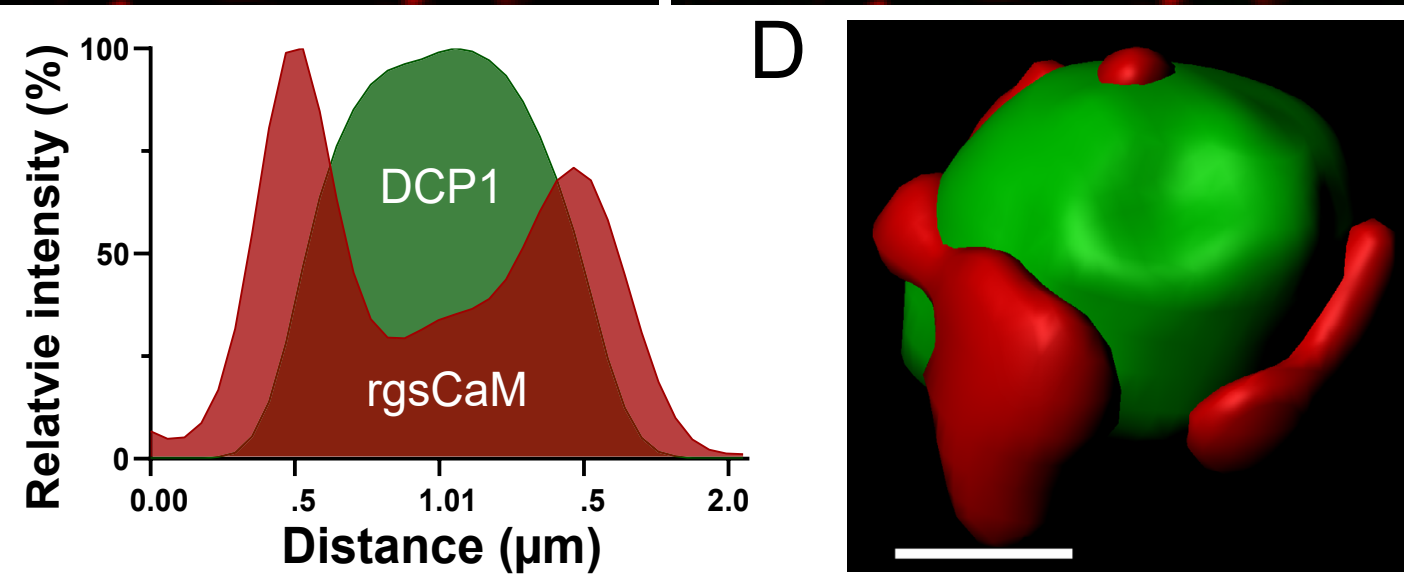

Fig 5. High resolution model of rgsCaM and DCP1 co-localization. A) A higher resolution two-dimensional projection from 27 optical sections $(0.3 \mu \mathrm{m}$ step size $)$ of a representative DCP1 processing body structure from a $N$. benthamiana cell co-transfected with DCP1-CFP and rgsCaM-YFP as described in Fig. 4. Leaf sections were subjected to submergence-induced hypoxia before imaging. The image was subjected to standard deconvolution and a smoothing filter was applied in Leica LasX software. Scale bars: top panel is $5 \mu \mathrm{m}$, bottom panel is $1 \mu \mathrm{m}$. B) A line profile for the fluorescence intensities through the dashed line shown in panel A. C) 3D model constructed from the dataset shown in A using the Imaris Software (see also Fig. S2). Scale bar is $0.5 \mu \mathrm{m}$. 

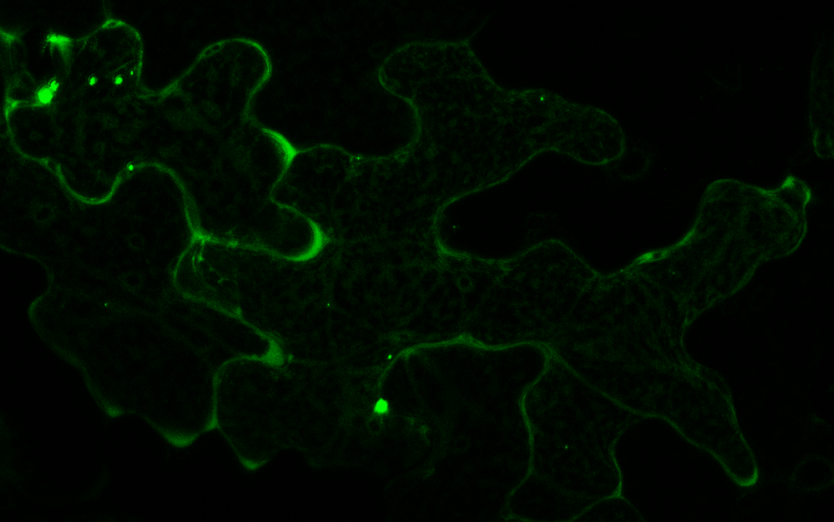

\section{$95 \mathrm{~min}$}

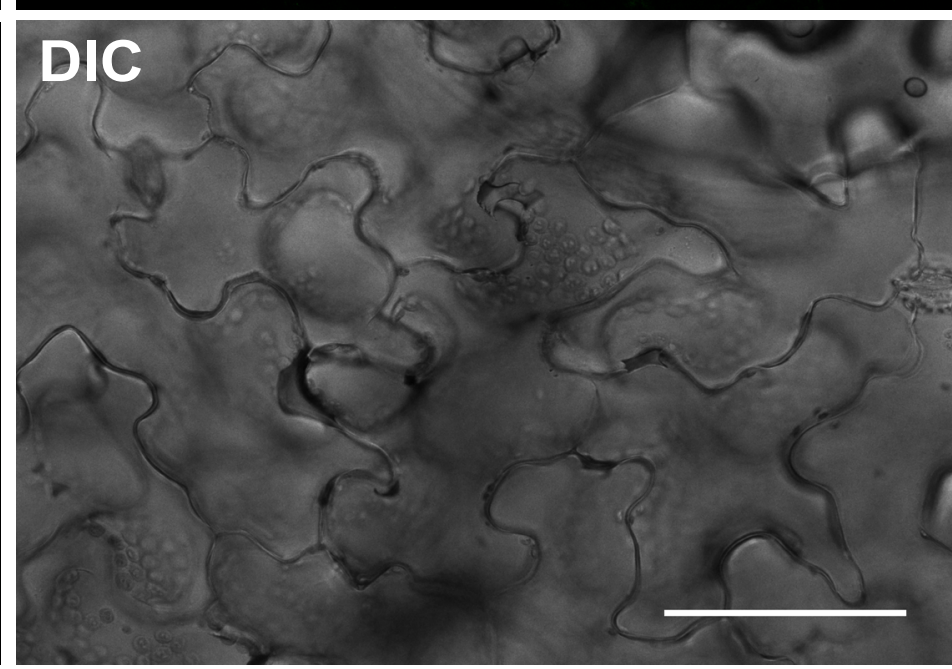

Fig 6. Time course of formation of SGS3-CFP cytosolic foci in response to submergence-induced hypoxia. $N$. benthamiana leaves were transfected with a SGS3-CFP construct by Agrobacterium infiltration and was imaged over the indicated times after submergence. Scale bar is $50 \mu \mathrm{m}$. 

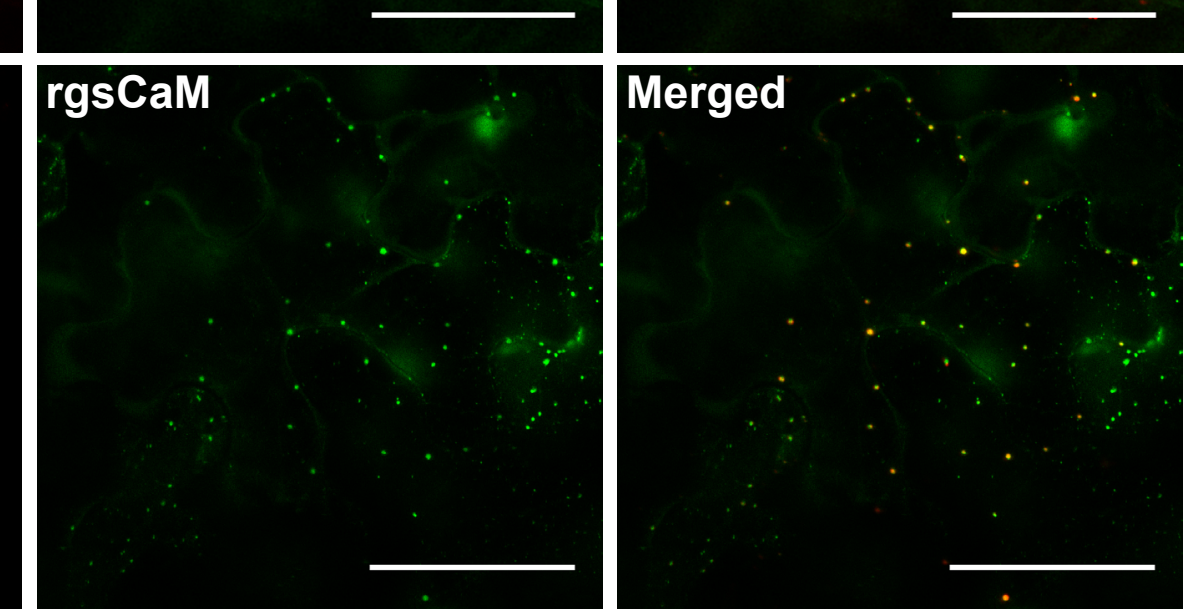

\section{DIC}

Fig 7. SGS3 and rgsCaM co-localize into cytosolic granule-like structures during hypoxia with different kinetics. N. benthamiana leaves were co-transfected with constructs that express rgsCaM-YFP and the stress granule marker SGS3-CFP and leaf sections were subjected to hypoxia as in Fig. 6 . Images are 2D projections of 6 optical sections with a step size of $2 \mu \mathrm{m}$ collected at $30 \mathrm{~min}$ and $90 \mathrm{~min}$ after submergence. Scale bars are $50 \mu \mathrm{m}$. 
B RgsCaM

\section{SGS3}

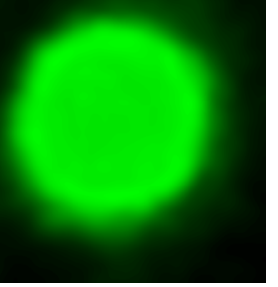

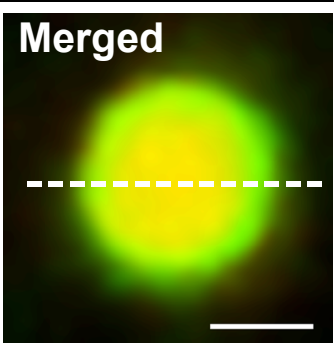

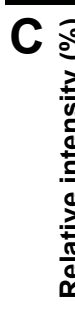

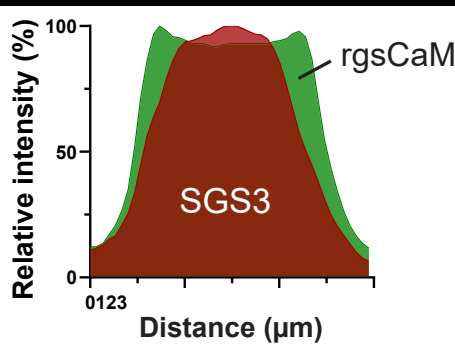

Fig 8. Co-localization of rgsCaM-CFP and SGS3-YFP. A) Leaves of N. benthamiana were co-transfected with constructs that express rgsCaM-YFP and SGS3-CFP, and leaf sections were subjected to submergence-induced hypoxia. Scale bar is 30 $\mu \mathrm{m}$. B) Enlargement of the structure in A marked with the arrowhead. Smooth rendering was applied in LAS X and deconvolution was performed with the Leica Lightning system. Scale bar is $1 \mu \mathrm{m}$. C) Line plot profile of fluorescence intensities through the dashed line in B. 


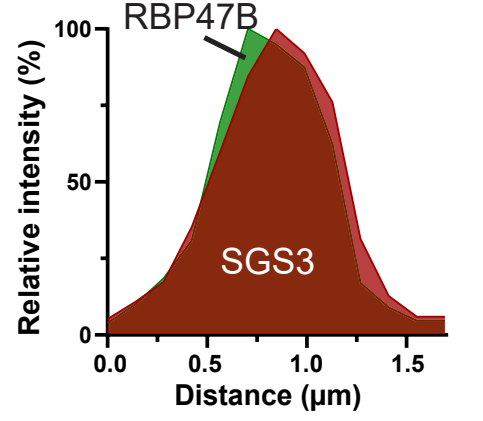

\section{SGS3}

\section{B RBP47B SGS3}
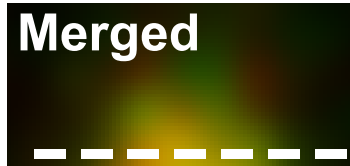

D SGS3

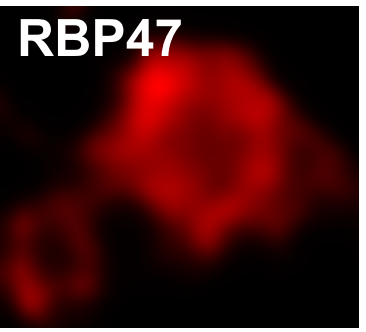

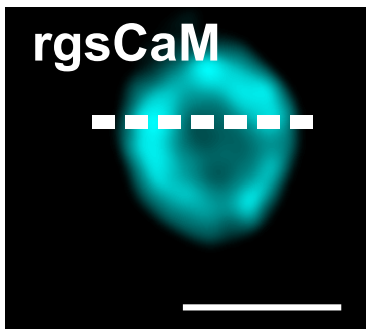
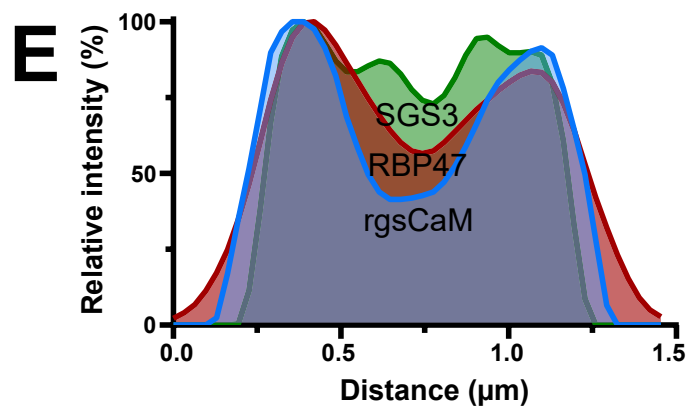

Fig 9. SGS3 co-localization with stress granule marker RBP47B N. benthamiana cells. A) Leaves of $N$. benthamiana were co-transfected with constructs that express SGS3-YFP and the stress granule marker RBP47B-CFP and leaf sections were subjected to hypoxia. Scale bar is $10 \mu \mathrm{m}$. B) Magnification of the granule marked by the arrowhead in A, with a smooth rendering applied in Leica LAS X. Scale bar is $0.5 \mu \mathrm{m}$. C) Line plot profile though the dashed line in B. D) Triple localization images from $N$. benthamiana leaf epidermal cells co-transfected with SGS3-CFP, RBP47B-RFP and rgsCaM-YFP. Images were taken $5 \mathrm{hr}$ after slide preparation and are 2D projection of 4 optical sections with a Z-step size of $0.13 \mu \mathrm{m}$. Scale bar is $1 \mu \mathrm{m}$. The Lightning deconvolution system was used and an additional smoothing filter was applied in LAS X. E) Line plot through the dashed line in D. 

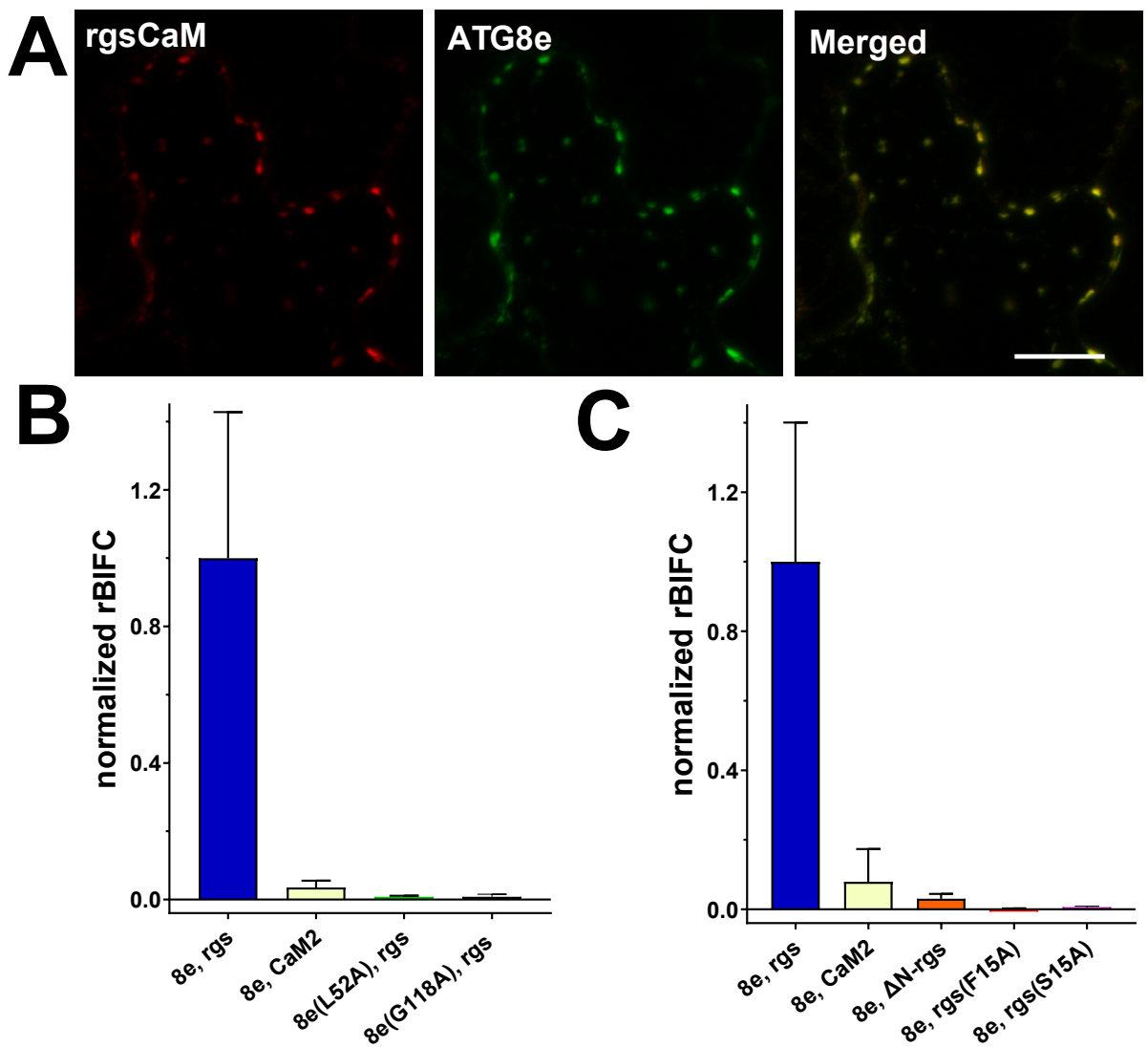

Fig 10. Co-localization and bimolecular fluorescence complementation analysis rgs CaM and ATG8e. A) Coexpression of rgsCaM-CFP and YFP-ATG8e in N. benthamiana leaves. Images were taken from leaf sections after submergence-induced hypoxia. Scale bars is $20 \mu \mathrm{m}$. B) Ratiometric BiFC quantification from $N$. benthamiana cells transfected with $\mathrm{pBiFC}$ constructs expressing the following protein pairs (see Materials and Methods for details): ATG8e, rgsCaM; ATG8e, AtCaM2; ATG8e, $\triangle \mathrm{N}$-rgsCaM; ATG8e, rgsCaM F15A; ATG8e, rgsCaM S16A. C) Ratiometric BiFC quantification from $N$. benthamiana cells transfected with pBiFC constructs done as in panel $B$, except with the following protein pairs: ATG8e, rgsCaM; ATG8e, AtCaM2; ATG8e L52A, rgsCaM; ATG8e G118A, rgsCaM. In both B and C, $\mathrm{BiFC}$ signals were standardized as a ratio to constitutive mRFP1 signals and were normalized to the highest value, which was the rgsCaM, ATG8e BiFC pair. The data represent the values from four biological replicates (20 to 30 cells). Error bars show standard deviation. Different letters over histograms represent statistically significant differences ( $p$ values $<0.0001$ ) based on One-way ANOVA analysis with multiple comparisons. 

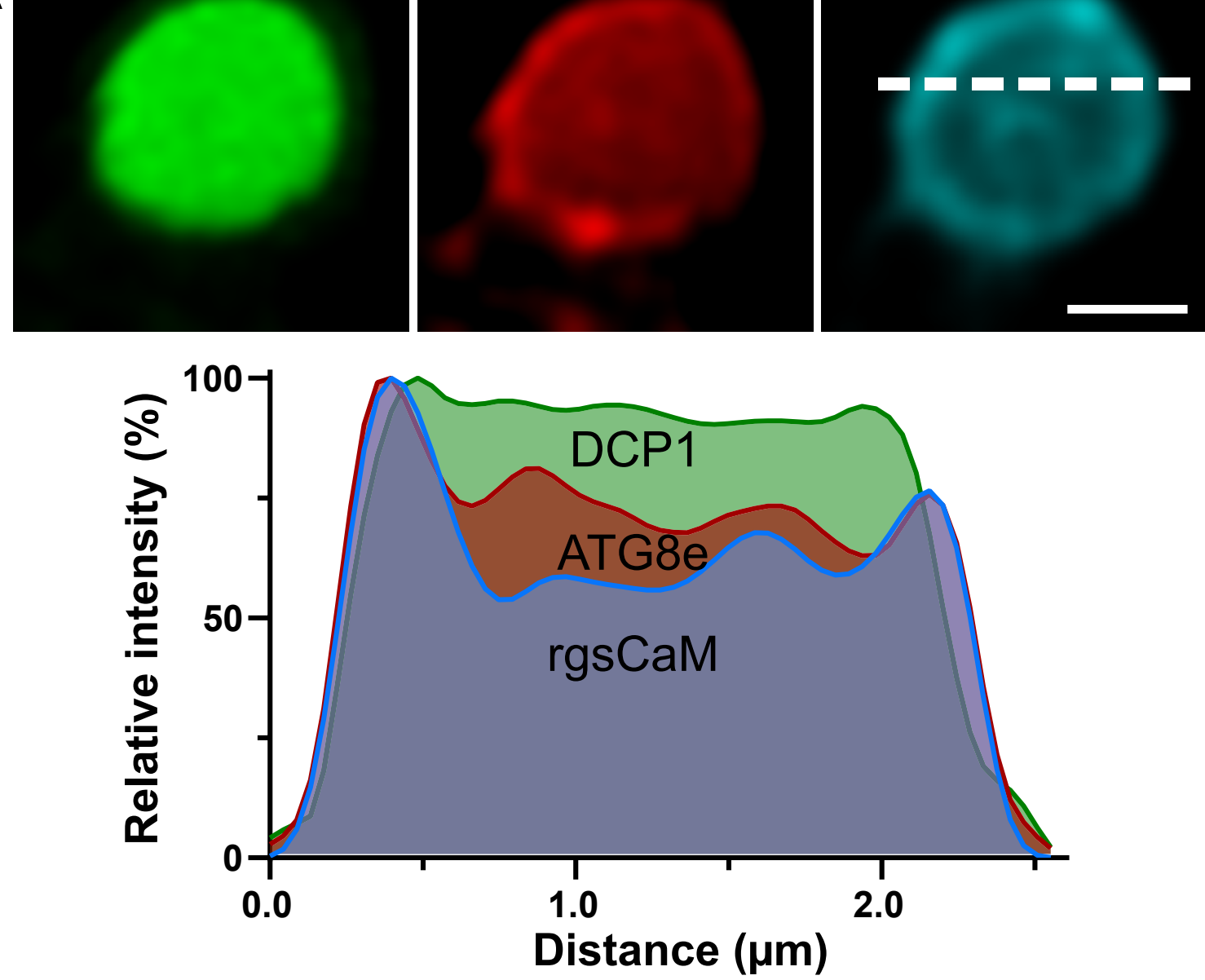

Fig. 11 Triple co-localization analysis of rgsCaM, ATG8e, and the PB marker DCP1 in hypoxic $\boldsymbol{N}$. benthamiana leaves. A) Co-localization of DCP1-CFP, YFP-ATG8e and rgsCaM-mScarlet transfected $N$. benthamiana leaves after submergence-induced hypoxia. Images are a 2D projection of 9 optical sections with a Z-step size of $0.2 \mu \mathrm{m}$. The scale bar is $1 \mu \mathrm{m}$. These images were generated using the Lightning deconvolution system. The smooth rendering filter was applied in LAS X. B) Line plot through the dashed line in panel A generated from the unfiltered data. 


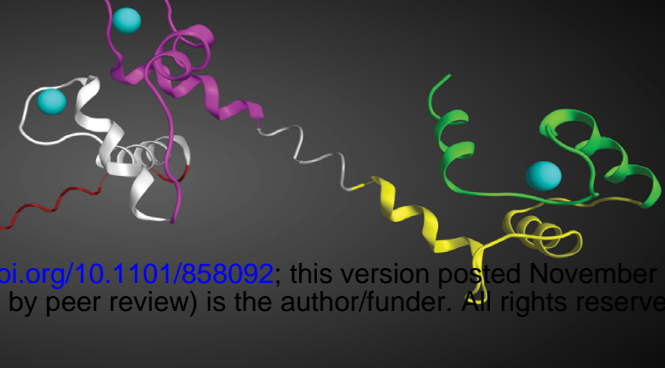

\section{ATG8}

autophagosome

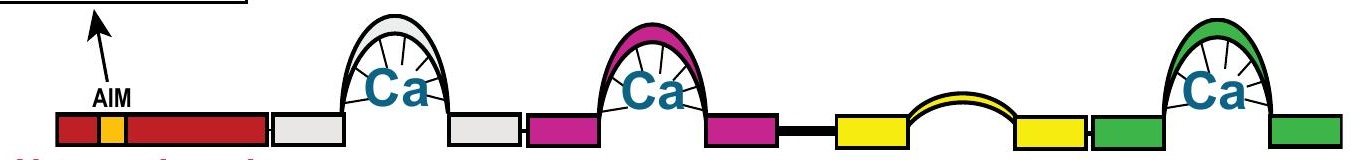

N-term domain

1st EF hand lobe 47-119

2nd EF hand lobe 128-190

1-46
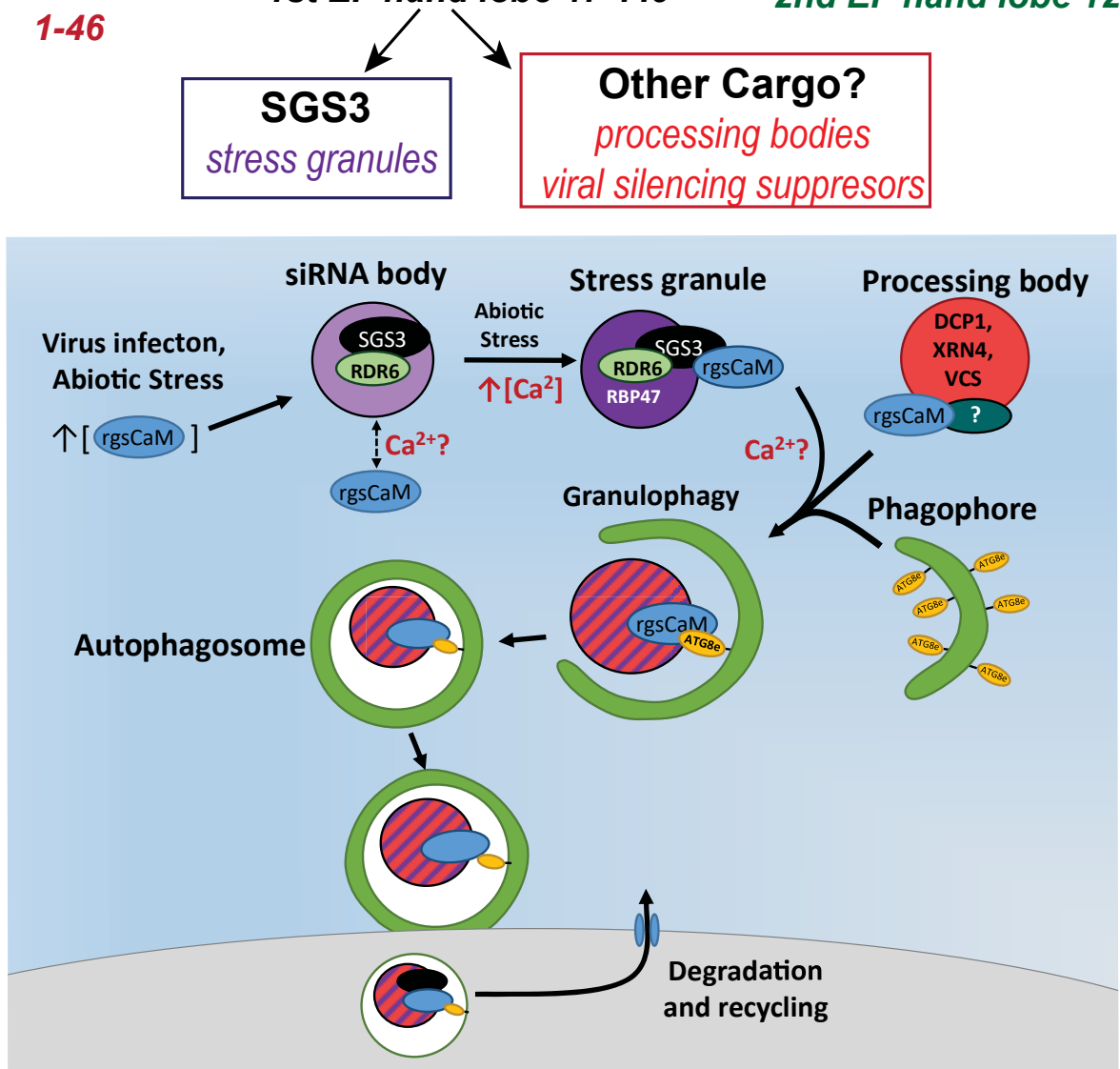

Fig. 12 Model for rgsCaM as a regulator of autophagic turnover of SGS3 and other cargo. A) Top panel, domain model for rgsCaM based on homology modeling with a calcium-calmodulin template (pdb 1cll). The bilobal structure with EF hands I (white) and II (magenta) comprising the $1^{\text {st }}$ calcium binding lobe (residues 47-119) and EF hands III (yellow) and IV (green) comprising the $2^{\text {nd }}$ calcium binding lobe. The $\mathrm{N}$-terminal domain is simply shown as an extended structure with the predicted AIM domain residues shown in space filled representation. Bottom panel, diagram showing the predicted sites of binding of rgsCaM to ATG8, as well as to SGS3 and other cargo (PB and viral suppressors of gene silencing). B) Model for rgsCaM mediated turnover of SGS3, RNP granules, and other cargo. RgsCaM and rgsCaM-like proteins (e.g., CML38) are induced during viral infection and selective abiotic stresses (e.g., hypoxia). Suppressor of gene silencing 3 (SGS3) resides in siRNA bodies as well as stress granule structures in complex with RNA-dependent RNA-polymerase 6 (RDR6). SGS3 recruits cytosolic rgsCaM to stress granules, and mediates the turnover (and suppression of secondary siRNA silencing) through targeting of SGS3 granules to autophagosomes (granulophagy) by rgsCaM interaction with ATG8. RgsCaM also interacts with processing bodies through interaction with an unknown PB protein target. In addition to degradation of the SGS3/ RDR6-mediated siRNA production machinery, autophagic degradation of mRNPs can recycle material during biotic and abiotic stress, and may control mRNA homeostasis and expression. 


\section{Parsed Citations}

Anandalakshmi R, Marathe R, Ge X, Herr JM, Jr., Mau C, Mallory A, Pruss G, Bowman L, Vance VB (2000) Acalmodulin-related protein that suppresses posttranscriptional gene silencing in plants. Science 290: 142-144

Pubmed: Author and Title

Google Scholar: Author Only Title Only Author and Title

Anderson P, Kedersha N (2008) Stress granules: the Tao of RNAtriage. Trends Biochem Sci 33: 141-150

Pubmed: Author and Title

Google Scholar: Author Only Title Only Author and Title

Bindels DS, Haarbosch L, van Weeren L, Postma M, Wiese KE, Mastop M, Aumonier S, Gotthard G, Royant A, Hink MA, Gadella TW, Jr. (2017) mScarlet: a bright monomeric red fluorescent protein for cellular imaging. Nat Methods 14: 53-56

Pubmed: Author and Title

Google Scholar: Author Only Title Only Author and Title

Birgisdottir AB, Lamark T, Johansen T (2013) The LIR motif - crucial for selective autophagy. J Cell Sci 126: $3237-3247$

Pubmed: Author and Title

Google Scholar: Author Only Title Only Author and Title

Bolte S, Cordelieres FP (2006) Aguided tour into subcellular colocalization analysis in light microscopy. J Microsc 224: 213-232

Pubmed: Author and Title

Google Scholar: Author Only Title Only Author and Title

Branco-Price C, Kaiser KA, Jang CJ, Larive CK, Bailey-Serres J (2008) Selective mRNA translation coordinates energetic and metabolic adjustments to cellular oxygen deprivation and reoxygenation in Arabidopsis thaliana. Plant $\mathrm{J}$ 56: $743-755$

Pubmed: Author and Title

Google Scholar: Author Only Title Only Author and Title

Brunkard JO, Burch-Smith TM, Runkel AM, Zambryski P (2015) Investigating Plasmodesmata Genetics with Virus-Induced Gene Silencing and an Agrobacterium-Mediated GFP Movement Assay. Plasmodesmata: Methods and Protocols 1217: 185-198

Pubmed: Author and Title

Google Scholar: Author Only Title Only Author and Title

Buchan JR (2014) mRNP granules. Assembly, function, and connections with disease. RNABiol 11: $1019-1030$

Pubmed: Author and Title

Google Scholar: Author Only Title Only Author and Title

Buchan JR, Kolaitis RM, Taylor JP, Parker R (2013) Eukaryotic stress granules are cleared by autophagy and Cdc48/VCP function. Cell 153: 1461-1474

Pubmed: Author and Title

Google Scholar: Author Only Title Only Author and Title

Chantarachot T, Bailey-Serres J (2018) Polysomes, Stress Granules, and Processing Bodies: ADynamic Triumvirate Controlling Cytoplasmic mRNA Fate and Function. Plant Physiol 176: 254-269

Pubmed: Author and Title

Google Scholar: Author Only Title Only Author and Title

Chen L, Liao B, Qi H, Xie LJ, Huang L, Tan WJ, Zhai N, Yuan LB, Zhou Y, Yu LJ, Chen QF, Shu W, Xiao S (2015) Autophagy contributes to regulation of the hypoxia response during submergence in Arabidopsis thaliana. Autophagy 11: 2233-2246

Pubmed: Author and Title

Google Scholar: Author Only Title Only Author and Title

Cheng X, Wang A(2017) The Potyvirus Silencing Suppressor Protein VPg Mediates Degradation of SGS3 via Ubiquitination and Autophagy Pathways. J Virol 91

Pubmed: Author and Title

Google Scholar: Author Only Title Only Author and Title

Chernov KG, Barbet A Hamon L, Ovchinnikov LP, Curmi PA, Pastre D (2009) Role of microtubules in stress granule assembly: microtubule dynamical instability favors the formation of micrometric stress granules in cells. J Biol Chem 284: 36569-36580

Pubmed: Author and Title

Google Scholar: Author Only Title Only Author and Title

Chitiprolu M, Jagow C, Tremblay V, Bondy-Chorney E, Paris G, Savard A, Palidwor G, Barry FA, Znman L, Keith J, Rogaeva E, Robertson J, Lavallee-Adam M, Woulfe J, Couture JF, Cote J, Gibbings D (2018) Acomplex of C9ORF72 and p62 uses arginine methylation to eliminate stress granules by autophagy. Nat Commun 9: 2794

Pubmed: Author and Title

Google Scholar: Author Only Title Only Author and Title

Contento AL, Xiong Y, Bassham DC (2005) Visualization of autophagy in Arabidopsis using the fluorescent dye monodansylcadaverine and a GFP-AtATG8e fusion protein. Plant J 42: 598-608

Pubmed: Author and Title

Google Scholar: Author Only Title Only Author and Title 
Dunn KW, Kamocka MM, McDonald JH (2011) A practical guide to evaluating colocalization in biological microscopy. Am J Physiol Cell Physiol 300: C723-742

Pubmed: Author and Title

Google Scholar: Author Only Title Only Author and Title

Elmayan T, Adenot X, Gissot L, Lauressergues D, Gy I, Vaucheret H (2009) A neomorphic sgs3 allele stabilizing miRNA cleavage products reveals that SGS3 acts as a homodimer. FEBS J 276: 835-844

Pubmed: Author and Title

Google Scholar: Author Only Title Only Author and Title

Endres MW, Gregory BD, Gao Z, Foreman AW, Mlotshwa S, Ge X, Pruss GJ, Ecker JR, Bowman LH, Vance V (2010) Two plant viral suppressors of silencing require the ethylene-inducible host transcription factor RAV2 to block RNA silencing. PLoS Pathog 6: e1000729

Pubmed: Author and Title

Google Scholar: Author Only Title Only Author and Title

Frankel LB, Lubas M, Lund AH (2017) Emerging connections between RNA and autophagy. Autophagy 13: 3-23

Pubmed: Author and Title

Google Scholar: Author Only Title Only Author and Title

Frisch DA, Harris-Haller LW, Yokubaitis NT, Thomas TL, Hardin SH, Hall TC (1995) Complete sequence of the binary vector Bin 19.

Plant Mol Biol 27: 405-409

Pubmed: Author and Title

Google Scholar: Author Only Title Only Author and Title

Fukunaga R, Doudna JA(2009) dsRNA with 5' overhangs contributes to endogenous and antiviral RNA silencing pathways in plants. EMBO J 28: 545-555

Pubmed: Author and Title

Google Scholar: Author Only Title Only Author and Title

Grefen C, Blatt MR (2012) A2in1 cloning system enables ratiometric bimolecular fluorescence complementation (rBiFC).

Biotechniques 53: 311-314

Pubmed: Author and Title

Google Scholar: Author Only Title Only Author and Title

Gutierrez-Beltran E, Moschou PN, Smertenko AP, Bozhkov PV (2015) Tudor staphylococcal nuclease links formation of stress granules and processing bodies with mRNA catabolism in Arabidopsis. Plant Cell 27: 926-943

Pubmed: Author and Title

Google Scholar: Author Only Title Only Author and Title

Hafren A, Lohmus A, Makinen K (2015) Formation of Potato Virus AInduced RNA Granules and Viral Translation Are Interrelated

Processes Required for Optimal Virus Accumulation. PLoS Pathog 11: e1005314

Pubmed: Author and Title

Google Scholar: Author Only Title Only Author and Title

Hafren A, Ustun S, Hochmuth A, Svenning S, Johansen T, Hofius D (2018) Turnip Mosaic Virus Counteracts Selective Autophagy of the Viral Silencing Suppressor HCpro. Plant Physiol 176: 649-662

Pubmed: Author and Title

Google Scholar: Author Only Title Only Author and Title

Hofgen R, Willmitzer L (1988) Storage of competent cells for Agrobacterium transformation. Nucleic Acids Res 16: 9877

Pubmed: Author and Title

Google Scholar: Author Only Title Only Author and Title

Ivanov P, Kedersha N, Anderson P (2019) Stress Granules and Processing Bodies in Translational Control. Cold Spring Harb Perspect Biol 11

Pubmed: Author and Title

Google Scholar: Author Only Title Only Author and Title

Jain S, Wheeler JR, Walters RW, Agrawal A, Barsic A, Parker R (2016) ATPase-Modulated Stress Granules Contain a Diverse Proteome and Substructure. Cell 164: 487-498

Pubmed: Author and Title

Google Scholar: Author Only Title Only Author and Title

Johansen T, Lamark T (2019) Selective Autophagy: ATG8 Family Proteins, LIR Motifs and Cargo Receptors. J Mol Biol

Pubmed: Author and Title

Google Scholar: Author Only Title Only Author and Title

Jones H (1995) Plant gene transfer and expression protocols. Humana Press, Totowa, N.J.

Pubmed: Author and Title

Google Scholar: Author Only Title Only Author and Title

Jouannet V, Moreno AB, Elmayan T, Vaucheret H, Crespi MD, Maizel A(2012) Cytoplasmic Arabidopsis AGO7 accumulates in membrane-associated siRNA bodies and is required for ta-siRNA biogenesis. EMBO J 31: 1704-1713

Pubmed: Author and Title 
Google Scholar: Author Only Title Only Author and Title

Karimi M, De Meyer B, Hilson P (2005) Modular cloning in plant cells. Trends Plant Sci 10: 103-105

Pubmed: Author and Title

Google Scholar: Author Only Title Only Author and Title

Kedersha N, Ivanov P, Anderson P (2013) Stress granules and cell signaling: more than just a passing phase? Trends Biochem Sci 38: 494-506

Pubmed: Author and Title

Google Scholar: Author Only Title Only Author and Title

Kedersha N, Stoecklin G, Ayodele M, Yacono P, Lykke-Andersen J, Fritzler MJ, Scheuner D, Kaufman RJ, Golan DE, Anderson P (2005)

Stress granules and processing bodies are dynamically linked sites of mRNP remodeling. J Cell Biol 169: $871-884$

Pubmed: Author and Title

Google Scholar: Author Only Title Only Author and Title

Kellner R, De la Concepcion JC, Maqbool A, Kamoun S, Dagdas YF (2017) ATG8 Expansion: ADriver of Selective Autophagy

Diversification? Trends Plant Sci 22: 204-214

Pubmed: Author and Title

Google Scholar: Author Only Title Only Author and Title

Kodama Y (2016) Time Gating of Chloroplast Autofluorescence Alows Clearer Fluorescence Imaging In Planta. PLoS One 11: e0152484

Pubmed: Author and Title

Google Scholar: Author Only Title Only Author and Title

Koncz C, Schell J (1986) The Promoter of TI-DNA Gene 5 Controls the Tissue-Specific Expression of Chimeric Genes Carried by a

Novel Type of Agrobacterium Binary Vector. Molecular \& General Genetics 204: 383-396

Pubmed: Author and Title

Google Scholar: Author Only Title Only Author and Title

Krisenko MO, Higgins RL, Ghosh S, Zhou Q, Trybula JS, Wang WH, Geahlen RL (2015) Syk Is Recruited to Stress Granules and

Promotes Their Clearance through Autophagy. J Biol Chem 290: 27803-27815

Pubmed: Author and Title

Google Scholar: Author Only Title Only Author and Title

Kumakura N, Takeda A, Fujioka Y, Motose H, Takano R, Watanabe Y (2009) SGS3 and RDR6 interact and colocalize in cytoplasmic SGS3/RDR6-bodies. Febs Letters 583: 1261-1266

Pubmed: Author and Title

Google Scholar: Author Only Title Only Author and Title

Li F, Huang C, Li Z, Zhou X (2014) Suppression of RNA silencing by a plant DNAvirus satellite requires a host calmodulin-like protein to repress RDR6 expression. PLoS Pathog 10: e1003921

Pubmed: $\underline{\text { Author and Title }}$

Google Scholar: Author Only Title Only Author and Title

Li F, Wang Y, Zhou X (2017) SGS3 Cooperates with RDR6 in Triggering Geminivirus-Induced Gene Silencing and in Suppressing Geminivirus Infection in Nicotiana Benthamiana. Viruses 9

Pubmed: Author and Title

Google Scholar: Author Only Title Only Author and Title

Li F, Zhao N, Li Z, Xu X, Wang Y, Yang X, Liu SS, Wang A, Zhou X (2017) A calmodulin-like protein suppresses RNA silencing and promotes geminivirus infection by degrading SGS3 via the autophagy pathway in Nicotiana benthamiana. PLoS Pathog 13: e1006213

Pubmed: Author and Title

Google Scholar: Author Only Title Only Author and Title

Lokdarshi A Conner WC, McClintock C, Li T, Roberts DM (2016) Arabidopsis CML38, a Calcium Sensor That Localizes to

Ribonucleoprotein Complexes under Hypoxia Stress. Plant Physiol 170: 1046-1059

Pubmed: Author and Title

Google Scholar: Author Only Title Only Author and Title

Loschi M, Leishman CC, Berardone N, Boccaccio GL (2009) Dynein and kinesin regulate stress-granule and P-body dynamics. J Cell Sci 122: 3973-3982

Pubmed: Author and Title

Google Scholar: Author Only Title Only Author and Title

Mallory AC, Ely L, Smith TH, Marathe R, Anandalakshmi R, Fagard M, Vaucheret H, Pruss G, Bowman L, Vance VB (2001) HC-Pro suppression of transgene silencing eliminates the small RNAs but not transgene methylation or the mobile signal. Plant Cell 13: 571583

Pubmed: Author and Title

Google Scholar: Author Only Title Only Author and Title

Manders EM, Stap J, Brakenhoff GJ, van Driel R, Aten JA(1992) Dynamics of three-dimensional replication patterns during the Sphase, analysed by double labelling of DNA and confocal microscopy. J Cell Sci 103 ( Pt 3): 857-862

Pubmed: Author and Title

Google Scholar: Author Only Title Only Author and Title 
Marshall RS, Vierstra RD (2018) Autophagy: The Master of Bulk and Selective Recycling. Annu Rev Plant Biol 69: 173-208

Pubmed: Author and Title

Google Scholar: Author Only Title Only Author and Title

Masson N, Keeley TP, Giuntoli B, White MD, Puerta ML, Perata P, Hopkinson RJ, Flashman E, Licausi F, Ratcliffe PJ (2019) Conserved $\mathrm{N}$-terminal cysteine dioxygenases transduce responses to hypoxia in animals and plants. Science 365: 65-69

Pubmed: Author and Title

Google Scholar: Author Only Title Only Author and Title

Michaeli S, Galili G, Genschik P, Fernie AR, Avin-Wittenberg T (2016) Autophagy in Plants--What's New on the Menu? Trends Plant Sci 21: 134-144

Pubmed: Author and Title

Google Scholar: Author Only Title Only Author and Title

Monahan Z, Shewmaker F, Pandey UB (2016) Stress granules at the intersection of autophagy and ALS. Brain Res 1649: 189-200

Pubmed: Author and Title

Google Scholar: Author Only Title Only Author and Title

Mourrain P, Beclin C, Elmayan T, Feuerbach F, Godon C, Morel JB, Jouette D, Lacombe AM, Nikic S, Picault N, Remoue K, Sanial M, Vo TA Vaucheret H (2000) Arabidopsis SGS2 and SGS3 genes are required for posttranscriptional gene silencing and natural virus resistance. Cell 101: 533-542

Pubmed: Author and Title

Google Scholar: Author Only Title Only Author and Title

Nakahara KS, Masuta C, Yamada S, Shimura H, Kashihara Y, Wada TS, Meguro A, Goto K, Tadamura K, Sueda K, Sekiguchi T, Shao J, Itchoda N, Matsumura T, Igarashi M, Ito K, Carthew RW, Uyeda I (2012) Tobacco calmodulin-like protein provides secondary defense by binding to and directing degradation of virus RNA silencing suppressors. Proc Natl Acad Sci U S A 109: 10113-10118

Pubmed: Author and Title

Google Scholar: Author Only Title Only Author and Title

Nakamura H, Shin MR, Fukagawa T, Arita M, Mikami T, Kodama H (2014) A tobacco calmodulin-related protein suppresses sense transgene-induced RNA silencing but not inverted repeat-induced RNA silencing. Plant Cell Tissue and Organ Culture 116: 47-53

Pubmed: Author and Title

Google Scholar: Author Only Title Only Author and Title

Noda NN, Ohsumi Y, Inagaki F (2010) Atg8-family interacting motif crucial for selective autophagy. FEBS Lett 584: 1379-1385

Pubmed: Author and Title

Google Scholar: Author Only Title Only Author and Title

OpenWetWare (2018) 'Round-the-horn site-directed mutagenesis. In,

Pubmed: Author and Title

Google Scholar: Author Only Title Only Author and Title

Protter DSW, Parker R (2016) Principles and Properties of Stress Granules. Trends Cell Biol 26: 668-679

Pubmed: Author and Title

Google Scholar: Author Only Title Only Author and Title

Schneider CA, Rasband WS, Eliceiri KW(2012) NIH Image to ImageJ: 25 years of image analysis. Nat Methods 9: 671-675

Pubmed: Author and Title

Google Scholar: Author Only Title Only Author and Title

Sorenson R, Bailey-Serres J (2014) Selective mRNA sequestration by OLIGOURIDYLATE-BINDING PROTEIN 1 contributes to translational control during hypoxia in Arabidopsis. Proceedings of the National Academy of Sciences of the United States of America 111: 2373-2378

Pubmed: Author and Title

Google Scholar: Author Only Title Only Author and Title

Stolz A, Ernst A, Dikic I (2014) Cargo recognition and trafficking in selective autophagy. Nat Cell Biol 16: 495-501

Pubmed: Author and Title

Google Scholar: Author Only Title Only Author and Title

Tadamura K, Nakahara KS, Masuta C, Uyeda I (2012) Wound-induced rgs-CaM gets ready for counterresponse to an early stage of viral infection. Plant Signal Behav 7: 1548-1551

Pubmed: Author and Title

Google Scholar: Author Only Title Only Author and Title

Vanderbeld B, Snedden WA(2007) Developmental and stimulus-induced expression patterns of Arabidopsis calmodulin-like genes CML37, CML38 and CML39. Plant Mol Biol 64: 683-697

Pubmed: Author and Title

Google Scholar: Author Only Title Only Author and Title

Weber C, Nover L, Fauth M (2008) Plant stress granules and mRNA processing bodies are distinct from heat stress granules. Plant $\mathrm{J}$ 56: 517-530

Pubmed: $\underline{\text { Author and Title }}$ 
Google Scholar: Author Only Title Only Author and Title

Xu GY, Rocha PS, Wang ML, Xu ML, Cui YC, Li LY, Zhu YX, Xia X (2011) Anovel rice calmodulin-like gene, OsMSR2, enhances drought and salt tolerance and increases ABA sensitivity in Arabidopsis. Planta 234: 47-59

Pubmed: Author and Title

Google Scholar: Author Only Title Only Author and Title

Yang X, Bassham DC (2015) New Insight into the Mechanism and Function of Autophagy in Plant Cells. Int Rev Cell Mol Biol 320: 1-40 Pubmed: Author and Title

Google Scholar: Author Only Title Only Author and Title

Yang X, Guo W, Li F, Sunter G, Zhou X (2019) Geminivirus-Associated Betasatellites: Exploiting Chinks in the Antiviral Arsenal of Plants. Trends Plant Sci 24: 519-529

Pubmed: Author and Title

Google Scholar: Author Only Title Only Author and Title

Yong Chung H, Lacatus G, Sunter G (2014) Geminivirus AL2 protein induces expression of, and interacts with, a calmodulin-like gene, an endogenous regulator of gene silencing. Virology 460-461: 108-118

Pubmed: Author and Title

Google Scholar: Author Only Title Only Author and Title

Zhan N, Wang C, Chen L, Yang H, Feng J, Gong X, Ren B, Wu R, Mu J, Li Y, Liu Z, Zhou Y, Peng J, Wang K, Huang X, Xiao S, Zuo J (2018) S-Nitrosylation Targets GSNO Reductase for Selective Autophagy during Hypoxia Responses in Plants. Mol Cell 71: 142-154 e146

Pubmed: Author and Title

Google Scholar: Author Only Title Only Author and Title 